Particle-size segregation and diffusive remixing in shallow granular avalanches

Gray, J.M.N.T. and Chugunov, V.A.

2006

MIMS EPrint: 2006.400

Manchester Institute for Mathematical Sciences

School of Mathematics

The University of Manchester

\footnotetext{
Reports available from: http://eprints.maths.manchester.ac.uk/

And by contacting: The MIMS Secretary

School of Mathematics

The University of Manchester

Manchester, M13 9PL, UK
} 


\title{
Particle-size segregation and diffusive remixing in shallow granular avalanches
}

\author{
By J. M. N. T. GRA Y ${ }^{1}$ AND V. A. CHUGUNOV 2 \\ ${ }^{1}$ School of Mathematics, University of Manchester, Manchester M13 9PL, UK \\ ${ }^{2}$ Centre for Information Technology, Kazan State University, Kazan, 420008, Russia
}

(Received 4 September 2005 and in revised form 8 June 2006)

Segregation and mixing of dissimilar grains is a problem in many industrial and pharmaceutical processes, as well as in hazardous geophysical flows, where the sizedistribution can have a major impact on the local rheology and the overall run-out. In this paper, a simple binary mixture theory is used to formulate a model for particlesize segregation and diffusive remixing of large and small particles in shallow gravitydriven free-surface flows. This builds on a recent theory for the process of kinetic sieving, which is the dominant mechanism for segregation in granular avalanches provided the density-ratio and the size-ratio of the particles are not too large. The resulting nonlinear parabolic segregation-remixing equation reduces to a quasi-linear hyperbolic equation in the no-remixing limit. It assumes that the bulk velocity is incompressible and that the bulk pressure is lithostatic, making it compatible with most theories used to compute the motion of shallow granular free-surface flows. In steady-state, the segregation-remixing equation reduces to a logistic type equation and the 'S'-shaped solutions are in very good agreement with existing particle dynamics simulations for both size and density segregation. Laterally uniform time-dependent solutions are constructed by mapping the segregation-remixing equation to Burgers equation and using the Cole-Hopf transformation to linearize the problem. It is then shown how solutions for arbitrary initial conditions can be constructed using standard methods. Three examples are investigated in which the initial concentration is (i) homogeneous, (ii) reverse graded with the coarse grains above the fines, and, (iii) normally graded with the fines above the coarse grains. Time-dependent twodimensional solutions are also constructed for plug-flow in a semi-infinite chute.

\section{Introduction}

Segregation of dissimilar grains is of great importance in the pharmaceutical, bulk chemical, food and agricultural industries. In some processes it is a desired and useful effect that can be used to separate particles from one another (e.g. Wills 1979). While in others it is undesired and it can be difficult to control (Johanson 1978). Ultimately this can significantly degrade the quality of the resulting products and in some circumstances it can have serious safety implications. There are a number of mechanisms for segregation of dissimilar grains including particle size (Bridgwater 1976), particle density (Drahun \& Bridgwater 1983), convection (Ehrichs et al. 1995), differential fluid drag (Zhang \& Reese 2000), inertia (Thomas 2000; Mobius et al. 2001) and collisional condensation (Jenkins 1998). This paper focuses on the competition between gravity-driven size-segregation by kinetic sieving (Bridgwater 1976; Savage \& Lun 1988) and diffusive remixing (e.g. Hsiau \& Hunt 1993; 


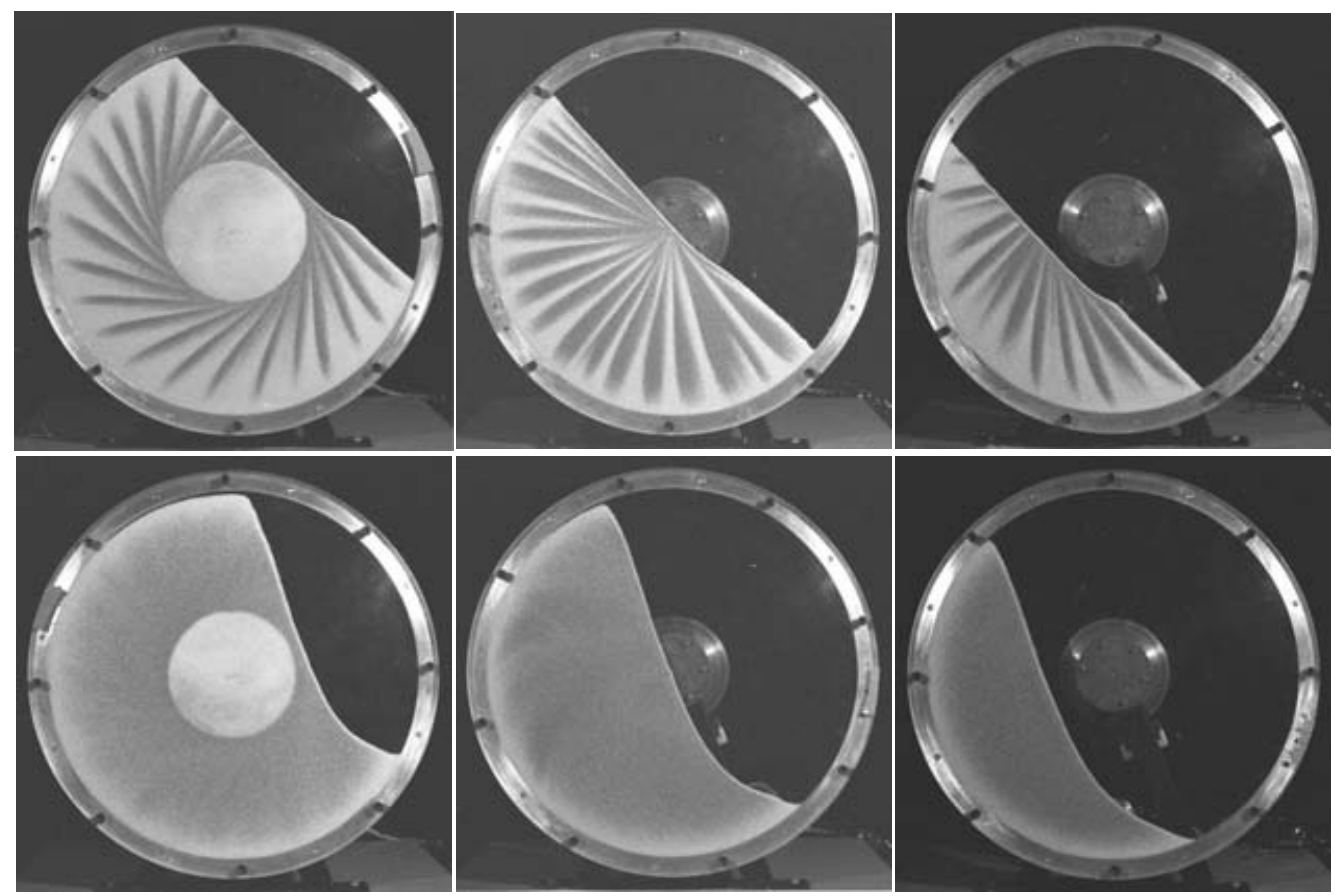

FIGURE 1. Patterns formed in a thin rotating drum partially filled with large (white) and small (grey) particles. All the segregation takes place in a very thin dynamic layer close to the free surface, which can be described as a granular avalanche. At low rotation rates, intermittent avalanches are released at the free surface (top). Segregation within the avalanche rapidly generates inversely graded layers that expand and are frozen into the deposit by the upslope propagation of a shock wave. The two-layer stripes laid down near the surface are then rotated and buried to create the patterns. At higher rotation rates (bottom) there is a steady-state avalanche near the free surface that is maintained by continuous erosion and deposition with the underlying solid rotating body of grains. In this higher energy flow, the diffusive remixing is considerably stronger, which is reflected in the distributed grading in the rotating deposit.

Savage \& Dai 1993; Dolgunin \& Ukolov 1995), which are the dominant processes in dense granular avalanches provided the density-ratio and the size-ratio of the particles are not too large (Drahun \& Bridgwater 1983; Thomas 2000).

The kinetic sieving mechanism is simple. As the grains avalanche downslope, there are fluctuations in the void space and the smaller particles are more likely to fall, under gravity, into gaps that open up beneath them, because they are more likely to fit into the available space than the coarse grains. The fine particles therefore percolate towards the bottom of the flow, and force imbalances squeeze the large particles towards the surface. Competing against this is diffusive remixing, which is caused by random motions of the particles as they collide and shear over one another. Dependent on the relative strengths of these two effects the particle size distribution can be either strongly or weakly reverse (or inversely) graded (Bagnold 1954; Middleton \& Hampton 1976) with the large particles above the fines. Figure 1 shows an example of this in a thin rotating drum that is partially filled with large white and small grey particles (Gray \& Hutter 1997; 1998). All the segregation takes place within a thin dynamic layer of grains close to the free surface, which can be described as a granular avalanche. At low rotation rates, avalanches are intermittently 
released close to the free surface and flow downslope. Kinetic sieving rapidly generates reverse graded layers within the avalanche with very high concentrations of coarse grains above very high concentrations of fines. When these are brought to rest by the upslope propagation of a granular bore (Gray \& Tai 1998; Gray, Tai \& Noelle 2003) the layers expand and are frozen into the deposit to create a two-layer reverse graded stripe at the free surface. These are subsequently buried and rotated with the solid body of material underneath to create the stripy patterns in the top panels of figure 1 . At higher rotation rates both the nature of the flow and the strength of the diffusive remixing change as shown in the lower panels of figure 1 . The avalanche is now in a steady state maintained by continuous erosion and deposition at its base (e.g. Gray 2001) and the diffusion is considerably stronger in this higher-energy flow. The reverse grading within the flowing avalanche is therefore weaker and this is reflected in the radial grading in the underlying rotating material. The sharp transitions at low rotation rates are now replaced by a much weaker distributed grading, which appears to be much darker than before. This is because the large white particles only show up clearly when they are close to being in a pure phase. The images therefore show that the grading is weak and the large particles are concentrated close to the drum wall, which is consistent with them being deposited out of the avalanche last. The small particles, which were at the bottom of the avalanche, are the first to be deposited and therefore lie closer to the centre of the drum.

There is now a considerable body of work on particle size segregation in kinetic binary mixtures (e.g. Jenkins \& Mancini 1987; Jenkins 1998; Khakhar, McCarthy \& Ottino 1999; Jenkins \& Yoon 2001; Xu, Louge \& Reeves 2003) with low solids volume fractions. These theories assume that the particles interact through binary collisions, and comparisons with particle dynamics simulations and microgravity experiments show that the theory works well for solids volume fractions $v<0.2$ (Khakhar $e$ al . 1999; Xu et al. 2003). At higher volume fractions kinetic theory can make reasonable predictions, but only when the effective granular temperature is used as a fitting parameter (Khakhar et al. 1999). However, Campbell (2006) points out that if the assumptions for kinetic theory break down for determining the granular temperature in dense flows, it is difficult to argue that the same assumptions work well in predicting segregation in the same flow.

A different theory is therefore required for segregation in granular avalanches, where the solids volume fractions are much higher $(v=0.49-0.64)$ and there are prolonged sliding and rolling contacts between the grains. There has been very little theoretical work in this area. Savage \& Lun (1988) developed the first theory using information entropy ideas to derive explicit formulae for the net percolation velocity of small particles. When this was substituted into the small particle mass balance, the theory was able to predict the formation of three steady-state concentration shocks that evolved and intersected with one another with increasing downstream distance. They observed similar shocks through the sidewall of their laboratory chute experiments and two photographs of them are reproduced by Thornton, Gray \& Hogg (2006). Savage \& Lun (1988) and Vallance \& Savage (2000) used a splitter-plate assembly to collect samples from different heights in the flow and at different downstream distances and were thus able to build up a detailed picture of the spatial particle size distribution. While this approach is not able to resolve the shocks, and there is some remixing close to the splitter plates, both Savage \& Lun (1988) and Vallance \& Savage (2000) found that their dilute theory was able to make accurate predictions of the particle-size distributions collected in the bins and this provides reasonable confirmation of the theory given their side-wall observations. 
Gray \& Thornton (2005) revisited Savage \& Lun's (1988) paper and used binary mixture theory to derive a simplified model. Assuming slow percolation, a linear drag between particles and that the fines carry less of the overburden pressure as they fall through the grain matrix, they used mass and momentum conservation to derive simple expressions for the large and small particle percolation velocities that satisfied a series of constraints. The simplest leading-order model that satisfied these constraints yielded a segregation flux that is dependent on the small particle concentration $\phi$ and is proportional to $\phi(1-\phi)$. If Savage \& Lun's (1988) model for the dilute small-particle concentration limit is rewritten in terms of the concentration $\phi$, rather than number density ratio $\eta$ and the particle diameter ratio $\sigma$, the two models have the same underlying $\phi(1-\phi)$ structure (Gray \& Thornton 2005). Furthermore, this structure still dominates in the non-dilute small-particle concentration limit. The experimental data of Savage \& Lun (1988) and Vallance \& Savage (2000) therefore provides considerable evidence for a segregation flux of this form. The key advantages of Gray \& Thornton's (2005) theory over that of Savage \& Lun (1988) is that: (i) the use of particle concentrations, rather than $\eta$ and $\sigma$, significantly simplifies the theory and (ii) gravity naturally enters into the segregation velocities through the normal momentum balance. The theory has been extended by Thornton et al. (2006) to include buoyancy effects through the introduction of an interstitial fluid and Gray $\&$ Thornton (2005) and Thornton et al. (2006) have developed robust shock-capturing numerical methods to solve general time-dependent problems without the need to explicitly track the position of the shocks. The insights that these have given have allowed exact solutions to be constructed for more general steady-state (Thornton et al. 2006) and fully time-dependent problems (Gray et al. 2006) in the absence of diffusive remixing effects.

Several authors have looked at the case when diffusive remixing effects are included in segregation models of this type. Dolgunin \& Ukolov (1995) and Dolgunin, Kudy \& Ukolov (1998) proposed a model for the segregation of different size and different density particles based on the observation that there could be no segregation when either of the constituents were in their pure phase, so that to leading order the segregation flux must be proportional to $\phi(1-\phi)$. The effect of remixing was modelled by the ad hoc introduction of a diffusion term and the associated diffusion coefficient was determined using considerations from kinetic theory. While using such a diffusion coefficient in the high solids volume fraction limit is itself debatable, Dolgunin \& Ukolov (1995) have confused the issue further by applying their theory to problems in which there is both a dense basal avalanche and a rarefied saltation layer. This seems to be pushing the model too far and we believe that the fundamental physical driving mechanisms for segregation are different in the dense and rarefied regimes. This has been recognized for some time in the geological literature where inverse-grading in pyroclastic flows is associated with dense basal grain flows (e.g. Middleton \& Hampton 1976) and normal-grading with sedimentation out of low-density turbulent suspension (e.g Branney \& Kokelaar 1992). A combination of these two effects can lead to double-segregation where the large particles are concentrated in the centre of a deposit.

For solids volume fractions $v<0.2$, Khakhar et al. (1999) and Xu et al. 2003 have shown that kinetic theory is perfectly good, which implicitly implies that Dolgunin \& Ukolov's (1995) model is too simple in this limit. However, Savage \& Lun's (1988) and Vallance \& Savage's (2000) data imply that the $\phi(1-\phi)$ flux model is a good one for particle-size segregation at high solids fractions, and Khakhar, McCarthy \& Ottino (1997) have also shown that Dolgunin \& Ukolov (1995) approach is very 
good at predicting density segregation at high solids volume fractions. The models of Savage \& Lun (1988), Dolgunin \& Ukolov's (1995) and Gray \& Thornton (2005) all have the same underlying $\phi(1-\phi)$ structure and appear to be good at predicting segregation in dense granular avalanches where there are multiple enduring frictional contacts between the grains. This paper extends Gray \& Thornton's (2005) rational derivation of the segregation equation to the case of segregation and remixing and investigates some elementary solutions.

\section{Governing equations}

\subsection{Mixture framework and conservation laws}

A simple binary mixture theory will be used to formulate a model for gravity-driven particle-size segregation and diffusive remixing in a granular material composed of 'large' and 'small' particles. This approach implicitly assumes that the interstitial pore space remains constant, on average, and that it can be subsumed into the volume fractions, $\phi^{l}$ and $\phi^{s}$, of large and small particles per unit mixture volume, respectively. The sum of these volume fractions is equal to unity

$$
\phi^{l}+\phi^{s}=1 \text {. }
$$

The superscripts $l$ and $s$ will be used throughout this paper to denote quantities relating to the large and small particles. The fundamental mixture postulate (e.g. Truesdell 1984; Morland 1992) states that every point in the mixture is simultaneously occupied by all of the constituents, so that overlapping partial densities, $\rho^{\mu}$, partial velocities, $\boldsymbol{u}^{\mu}$, and partial pressures, $p^{\mu}$, can be defined for each of the constituents $\mu=l, s$ per unit mixture volume. Each of the constituents satisfies individual mass and momentum conservation laws (e.g. Truesdell 1984; Morland 1992; Gray \& Svendsen 1997)

$$
\begin{gathered}
\frac{\partial \rho^{\mu}}{\partial t}+\nabla \cdot\left(\rho^{v} \boldsymbol{u}^{\nu}\right)=0 \quad(\mu=l, s), \\
\frac{\partial}{\partial t}\left(\rho^{\mu} \boldsymbol{u}^{\mu}\right)+\nabla \cdot\left(\rho^{\mu} \boldsymbol{u}^{\mu} \otimes \boldsymbol{u}^{\mu}\right)=-\nabla p^{\mu}+\rho^{\mu} \boldsymbol{g}+\boldsymbol{\beta}^{\mu} \quad(\mu=l, s),
\end{gathered}
$$

where $\otimes$ is the dyadic product, $\rho^{\mu} \boldsymbol{g}$ is the gravitational force and $\boldsymbol{\beta}^{\mu}$, is the force exerted on phase $v$ by the other constituent. Newton's third law implies that the interaction forces in a binary mixture are equal and opposite to one another, i.e. $\boldsymbol{\beta}^{l}=-\boldsymbol{\beta}^{s}$, and cancel out in the bulk mass and momentum balances, which are obtained by summing the conservation laws (2.2) and (2.3) over all constituents. It is useful to define the bulk density $\rho$, bulk velocity $\boldsymbol{u}$ and bulk pressure $p$ as

$$
\rho=\rho^{l}+\rho^{s}, \quad \rho \boldsymbol{u}=\rho^{l} \boldsymbol{u}^{l}+\rho^{s} \boldsymbol{u}^{s}, \quad p=p^{l}+p^{s} .
$$

A key feature of mixture theory is how partial quantities, defined per unit mixture volume, are related to measurable intrinsic quantities, defined per unit constituent volume. Morland (1992) showed that the partial and intrinsic densities are related by a linear volume fraction scaling, while the partial and intrinsic velocity fields are identical, i.e.

$$
\rho^{\mu}=\phi^{\mu} \rho^{\mu^{*}}, \quad \boldsymbol{u}^{\mu}=\boldsymbol{u}^{\mu^{*}},
$$

where the superscript $*$ denotes an intrinsic variable. The partial and intrinsic pressures can, however, be related by any functional form provided $(2.4 c)$ is not violated. 


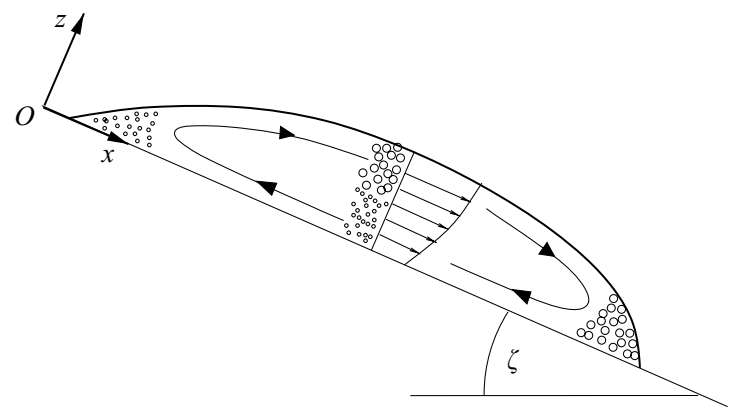

FIGURE 2. A sketch of the coordinate system $O x z$ and the particle size-distribution within an avalanche. The large arrows indicate that velocity shear leads to recirculation.

\subsection{Compatibility with existing avalanche models}

Following Gray \& Thornton (2005), a coordinate system $O x y z$ is defined, with the $x$-axis pointing down a chute inclined at an angle $\zeta$ to the horizontal, the $y$-axis across the chute and the $z$-axis as the upward pointing normal as shown in figure 2 . The constituent velocity $\boldsymbol{u}^{\mu}$ and the bulk velocity $\boldsymbol{u}$ have components $\left(u^{\mu}, v^{\mu}, w^{\mu}\right)$ and $(u, v, w)$ in each of these directions, respectively. The intrinsic densities of the large and small particles, $\rho^{l *}$ and $\rho^{s^{*}}$, are assumed to be equal to the same constant value. It follows from (2.1) and (2.4a), that the bulk density is also constant

$$
\rho=\rho^{l *}=\rho^{s *}=\text { const, }
$$

and, using $(2.4 b)$, that the bulk mass balance reduces to $\nabla \cdot \boldsymbol{u}=0$, which implies that the bulk velocity is incompressible. Note, that in this two-constituent formulation these densities are actually equal to the mean solids fraction multiplied by the bulk solid density. Assuming that the normal acceleration terms are negligible in (2.3), the sum of the normal components of the large and small particle momentum balances implies that the normal component of the bulk momentum balance is

$$
\frac{\partial p}{\partial z}=-\rho g \cos \zeta
$$

where $g$ is the coefficient of acceleration due to gravity. As $\rho$ is constant and the free-surface is traction free, this can be integrated through the avalanche depth $h$ to show that the bulk pressure is lithostatic

$$
p=\rho g(h-z) \cos \zeta .
$$

The segregation-remixing theory is therefore consistent with the two key assumptions made by most current avalanche models (e.g. Grigorian, Eglit \& Iakimov 1967; Kulikovskii \& Eglit 1973; Eglit 1983; Savage \& Hutter 1989; Iverson 1997; Gray, Wieland \& Hutter 1999; Iverson \& Denlinger 2001; Gray 2001; Gray et al. 2003) that the bulk velocity $\boldsymbol{u}$ is incompressible and the overburden pressure $p$ is lithostatic.

\subsection{The particle-size segregation and diffusive remixing model}

One of the key ideas behind Gray \& Thornton's (2005) particle-size segregation model was that as the small particles percolate downwards through the grain matrix, they carry less of the overburden pressure, and so, the large particles must carry proportionately more of the load. Gray \& Thornton (2005) introduced a new pressure scaling, in which the partial pressure was linearly related to the bulk pressure by a 
factor $f^{\mu}$

$$
p^{\mu}=f^{\mu} p \quad(\mu=l, s) .
$$

The factors $f^{\mu}$ determine how much of the overburden pressure is carried by each of the constituents. Often $f^{\mu}$ is assumed to be equal to $\phi^{\mu}$ in standard mixture theories, but here it is the perturbations away from $\phi^{\mu}$ that are crucial for the segregation. The functions $f^{\mu}$ satisfy three constraints; from the definition of the bulk pressure (2.4c) the factors $f^{\mu}$ must sum to unity

$$
f^{l}+f^{s}=1
$$

and when either one of the constituents is in a pure phase they must carry all of the load

$$
f^{\mu}=1 \text { when } \phi^{\mu}=1 \text { for } \mu=l, s .
$$

A wide class of functions satisfy these constraints, but this paper focuses on the model suggested by Gray \& Thornton (2005), where

$$
f^{l}=\phi^{l}+b \phi^{s} \phi^{l}, \quad f^{s}=\phi^{s}-b \phi^{s} \phi^{l},
$$

and $b$ is the magnitude of the non-dimensional perturbation away from $\phi^{\mu}$. Not only are these the simplest non-trivial functions that satisfy the constraints (2.10) and (2.11), but, as will be shown in $\S 2.4$, they lead to the same segregation flux function as used by Savage \& Lun (1988) and Dolgunin \& Ukolov (1995).

The second key assumption in Gray \& Thornton's (2005) size-segregation model was the form of the interaction drag $\boldsymbol{\beta}^{\mu}$. It consisted of a linear velocity-dependent drag and a grain-grain surface interaction force $p \nabla f^{\mu}$ that combined with the partial pressure gradient $-\nabla\left(f^{\mu} p\right)$ to leave a net contribution of $-f^{\mu} \nabla p$ in the constituent momentum balances. These terms are retained in the current model and an additional remixing force $-\rho d \nabla \phi^{\mu}$ (e.g. Bear 1972; Morland 1992) is added, that seeks to drive grains of phase $v$ towards areas of lower concentration. The interaction drag is therefore

$$
\boldsymbol{\beta}^{\mu}=p \nabla f^{\mu}-\rho^{\mu} c\left(\boldsymbol{u}^{\mu}-\boldsymbol{u}\right)-\rho d \nabla \phi^{\mu} \quad(\mu=l, s),
$$

where $c$ is the linear drag coefficient and $d$ determines the strength of the diffusive forces. Note, that the definitions (2.4) imply that (2.13) satisfies the constraint that $\boldsymbol{\beta}^{l}+\boldsymbol{\beta}^{s}=0$, so they cancel out in the bulk momentum balance.

Assuming that the acceleration terms are negligible in the normal component of the constituent momentum balances (2.3), and substituting the pressure scaling (2.9), the lithostatic pressure gradient (2.7) and the interaction drag (2.13), implies that the normal flux of particles $\mu$ is

$$
\phi^{\mu} w^{\mu}=\phi^{\mu} w+\left(f^{\mu}-\phi^{\mu}\right)(g / c) \cos \zeta-(d / c) \partial \phi^{\mu} / \partial z \quad(\mu=l, s) .
$$

The first term on the right-hand side transports the concentration $\phi^{\mu}$ with the bulk normal velocity $w$, the second term is responsible for segregation and the third term for remixing. Since (2.12) implies that $f^{l}-\phi^{l}$ is positive and $f^{s}-\phi^{s}$ is negative, the segregation terms drives an upward flux of large particles and a corresponding downward flux of fines. The remixing term, on the other hand, drives fluxes of large and small particles towards regions of lower concentration. Substituting (2.12) and dividing through by the volume fraction $\phi^{\mu}$ implies that the normal velocities are

$$
w^{l}=w+q \phi^{s}-D \frac{\partial}{\partial z}\left(\ln \phi^{l}\right), \quad w^{s}=w-q \phi^{l}-D \frac{\partial}{\partial z}\left(\ln \phi^{s}\right),
$$


where the mean segregation velocity $q$ and the diffusivity $D$ are

$$
q=(b / c) g \cos \zeta, \quad D=d / c .
$$

Although the theory introduces a pressure perturbation coefficient $b$, a linear drag coefficient $c$ and a diffusive force coefficient $d$, equation (2.16) shows that they appear as ratios, so there are in fact only two independent coefficients $q$ and $D$ in the theory. Physically, $q$ determines the maximum percolation velocity of the grains, while the diffusivity $D$ determines the strength of the remixing. The segregation velocity increases with the particle-size ratio, the strength of gravity and the degree of dilation/agitation, which is itself dependent on the local shear rate in these fluid-like high-density flows, while the diffusivity increases with agitation/shear-rate. Experiments suggest that the dependencies are non-trivial. For instance, Hajra \& Khakhar (2004) used rotating drum experiments, such as those illustrated in figure 1, to infer that even small size differences are sufficient to cause segregation, but once the size ratio reaches a critical value the driving force for segregation saturates. This is consistent with the notion that once sufficient dilatation has occurred for the grains to percolate freely, there is no further increase in the segregation velocity. One of the benefits of our theory is that it provides a natural way of introducing gravity into the segregation velocity. As opposed to the phenomenological theory of Dolgunin \& Ukolov (1995) and the statistical mechanics/information entropy approach of Savage \& Lun (1988), this automatically sets a direction for segregation and accurately reflects the gravity-driven nature of the kinetic sieving process. Unlike Savage \& Lun's model, our theory does not yield formulae for the other functional dependencies of the segregation velocity $q$ or the diffusivity $D$. In order to apply the model, either empirical laws for the dependencies must be determined, or, average values must be measured in a given experiment. Ultimately, it may also be possible to use particle-dynamics simulations or a modified statistical mechanics approach similar to Savage \& Lun (1988) to yield more detailed theoretical information about the appropriate functional forms.

The mathematical structure of the expressions for the segregation velocities in (2.15) imply that, in the absence of diffusion, the large particles will rise towards the surface until there are no more small particles to fall down through the gaps and a pure phase of coarse grains is formed. Conversely, the fines will percolate down until there are no more large grains to be levered upwards. The steady-state configuration in the absence of diffusion therefore consists of a pure layer of large particles on top of a pure layer of fines as observed in the experiments of Savage \& Lun (1988). The segregation-remixing model introduces a diffusive term into (2.14) that competes against particle-size segregation, resulting in a smoothly distributed inverse grading through the depth of the avalanche.

\subsection{The segregation-remixing equation}

The velocities induced by particle-size segregation and diffusive remixing are assumed to be of the same order of magnitude as the bulk normal velocity. Since the shallowness of the avalanche implies that the lateral velocity components are much larger than the normal velocity, it follows that, to leading order, the lateral components of the constituent velocities are equal to the bulk velocity

$$
u^{\mu}=u, \quad v^{\mu}=v \quad(\mu=l, s) .
$$

An equation for particle-size segregation and diffusive remixing is obtained by substituting the partial densities (2.5) and the constituent velocities (2.15) and (2.17) 
into the small particle mass balance (2.2) to give

$$
\frac{\partial \phi^{s}}{\partial t}+\frac{\partial}{\partial x}\left(\phi^{s} u\right)+\frac{\partial}{\partial y}\left(\phi^{s} v\right)+\frac{\partial}{\partial z}\left(\phi^{s} w\right)-\frac{\partial}{\partial z}\left(q \phi^{s} \phi^{l}\right)=\frac{\partial}{\partial z}\left(D \frac{\partial \phi^{s}}{\partial z}\right) .
$$

The first four terms sweep the local concentration along with the bulk flow, while the fifth term is responsible for particle-size segregation and the sixth for diffusive remixing.

Anticipating that this equation will be used in conjunction with existing avalanche models, it is non-dimensionalized by using the standard avalanche scalings. These assume that the avalanche thickness $H$ is much smaller than its length $L$, so that the aspect ratio $\varepsilon=H / L \ll 1$. Incompressibility then implies that typical downslope velocity magnitudes $U$ are much larger than the normal velocity which is of magnitude $H U / L$. The variables are therefore non-dimensionalized by the scalings

$$
(x, y, z)=L(\tilde{x}, \tilde{y}, \varepsilon \tilde{z}), \quad(u, v, w)=U(\tilde{u}, \tilde{v}, \varepsilon \tilde{w}), \quad t=(L / U) \tilde{t},
$$

where the tilde denotes a non-dimensional variable. Substituting these into (2.18) and dropping the tildes and the superscript $s$ implies that the non-dimensional segregation-remixing equation is

$$
\frac{\partial \phi}{\partial t}+\frac{\partial}{\partial x}(\phi u)+\frac{\partial}{\partial y}(\phi v)+\frac{\partial}{\partial z}(\phi w)-\frac{\partial}{\partial z}\left(S_{r} \phi(1-\phi)\right)=\frac{\partial}{\partial z}\left(D_{r} \frac{\partial \phi}{\partial z}\right),
$$

where the non-dimensional segregation and diffusive-remixing numbers

$$
S_{r}=\frac{q L}{H U}, \quad D_{r}=\frac{D L}{H^{2} U},
$$

control the length and time scales for segregation and remixing in avalanche flows. The theory therefore contains only two parameters. The segregation flux $\mathscr{F}=-S_{r} \phi(1-\phi)$ has the same structure as that proposed by Savage \& Lun (1988) and Dolgunin \& Ukolov (1995). The three models are therefore closely related and the experimental comparisons that they made provide considerable evidence that the simple form of the pressure perturbations in (2.12) is, in fact, a very good model. Savage \& Lun (1988) used splitter plates to collect samples from different heights and different positions in the flow and were thus able to build up a two-dimensional steady-state picture of the particle size distribution along the length of the chute. They showed that, as an avalanche with a homogenous inflow concentration moved down the chute, three concentration shocks were generated. Two of these were initiated at the release point, and separated material that was still close to the inflow concentration from pure coarse grains above and pure fines below. The third was created some way down the chute, once the flow had fully separated to create an inversely graded stratified layer. Exactly the same structure develops naturally in models using the segregation flux $\mathscr{F}=-S_{r} \phi(1-\phi)$ in the absence of diffusion.

\subsection{Boundary conditions}

Provided there is no erosion or deposition, the appropriate conditions at the surface and base of the flow are that there is no flux of small particles across the boundary. This can be expressed mathematically by using a limiting argument (e.g Chadwick 1976) at a surface of discontinuity to show that (2.20) implies that the jump must satisfy

$$
\llbracket \phi\left(\boldsymbol{u} \cdot \boldsymbol{n}-v_{n}\right) \rrbracket-\llbracket S_{r} \phi(1-\phi) \boldsymbol{k} \cdot \boldsymbol{n} \rrbracket=\llbracket D_{r}(\partial \phi / \partial z) \boldsymbol{k} \cdot \boldsymbol{n} \rrbracket,
$$


where the jump bracket $\llbracket f \rrbracket=f^{+}-f^{-}$is the difference of $f$ evaluated on the forward ' + ' side and rearward ' - ' side of the surface, $\boldsymbol{k}$ is the unit vector normal to the chute, $\boldsymbol{n}$ is the unit normal to the surface and $v_{n}$ is its normal speed of propagation. At the free surface $F=z-z_{s}(x, y, t) \equiv 0$, the normal $n=\nabla F /|\nabla F|$ and $v_{n}$ satisfies $\partial F / \partial t+v_{n}|\nabla F|=0$ (e.g. Sethian 1999). Since there are no small particles outside the avalanche, $\phi^{+} \equiv 0$, and the surface boundary condition reduces to

$$
-\phi\left[\frac{\partial z}{\partial t}+u \frac{\partial z}{\partial x}+v \frac{\partial z}{\partial y}-w\right]=S_{r} \phi(1-\phi)+D_{r} \frac{\partial \phi}{\partial z} \quad \text { on } \quad z=z_{s},
$$

where $\phi^{-}$is assumed to be equal to $\phi$. The square-bracketed term on the right-hand side of this equation is simply the kinematic boundary condition at the surface of the avalanche, which is identically zero (e.g. Gray et al. 1999, 2003). A similar argument holds at the base of the flow, $z=z_{b}(x, y, t)$. The surface and basal no flux conditions therefore reduce to

$$
S_{r} \phi(1-\phi)+D_{r} \frac{\partial \phi}{\partial z}=0 \quad \text { on } \quad z=z_{s}, z_{b} .
$$

This nonlinear boundary condition insulates the segregation and remixing taking place within the avalanche from the exterior.

\section{Steady-state solutions for uniform flows}

For steady-uniform flow of constant height, the scalings (2.19) imply that the non-dimensional thickness can without loss of generality be chosen to be unity. Assuming lateral uniformity in concentration and that the time-derivatives are zero, the segregation-remixing equation (2.20) and no normal flux boundary conditions (2.24) reduce to

$$
\begin{gathered}
-S_{r} \frac{\partial}{\partial z}(\phi(1-\phi))=D_{r} \frac{\partial^{2} \phi}{\partial z^{2}} \quad(0<z<1), \\
S_{r} \phi(1-\phi)+D_{r} \frac{\partial \phi}{\partial z}=0 \quad(z=0,1) .
\end{gathered}
$$

Integrating (3.1) with respect to $z$ implies that the small-particle normal flux, $S_{r} \phi(1-$ $\phi)+D_{r} \partial \phi / \partial z$, is equal to a constant throughout the flow. This has the same form as both the boundary conditions (3.2), which implies that the constant of integration is equal to zero. Note, that for a steady state to exist, the boundary fluxes must be equal to one another, as they are here. The resulting first-order ODE is a form of the logistic equation and can be integrated again by separation of variables. However, the boundary conditions (3.2) are not sufficient to determine the constant of integration and a further criterion must be imposed. The mean concentration in the layer $0 \leqslant z \leqslant 1$ is therefore assumed to be

$$
\phi_{m}=\int_{0}^{1} \phi \mathrm{d} z
$$

which implies that the steady-state concentration

$$
\phi_{s}=\frac{\left(1-\exp \left(-\phi_{m} / z_{0}\right)\right) \exp \left(\left(\phi_{m}-z\right) / z_{0}\right)}{1-\exp \left(-\left(1-\phi_{m}\right) / z_{0}\right)+\left(1-\exp \left(-\phi_{m} / z_{0}\right)\right) \exp \left(\left(\phi_{m}-z\right) / z_{0}\right)},
$$

where

$$
z_{0}=\frac{D_{r}}{S_{r}}=\frac{D}{H q}=\frac{d}{H b g \cos \zeta}
$$




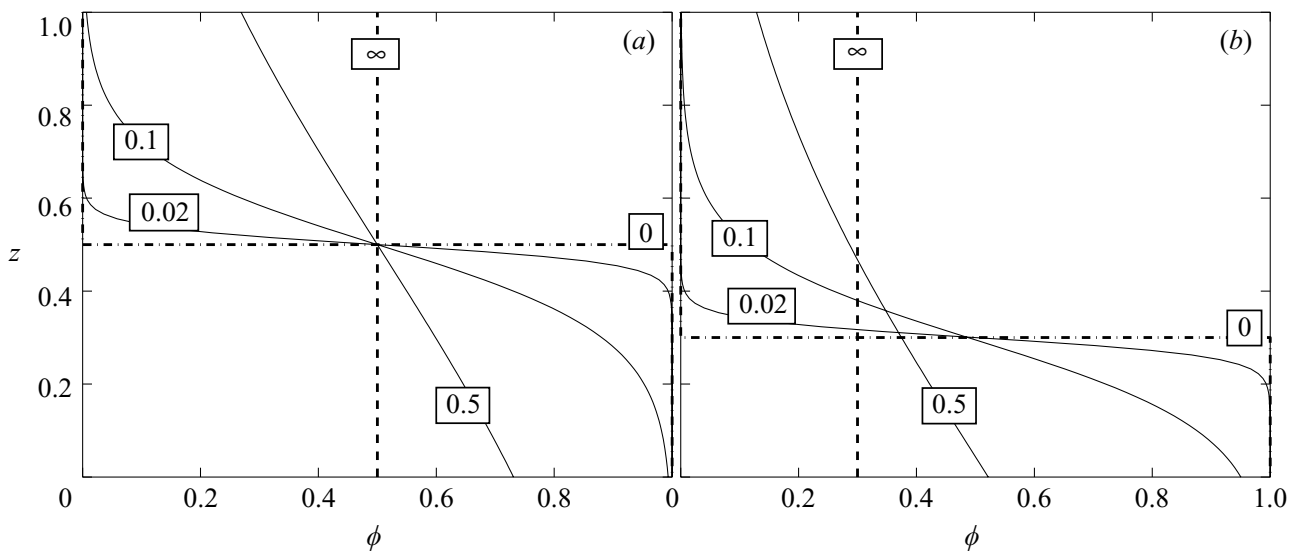

FiguRE 3. The one-dimensional steady-state small-particle concentration distribution $\phi$ as a function of avalanche depth $z$ is plotted for $z_{0}=0,0.02,0.1,0.5, \infty$ and for $(a) \phi_{m}=0.5$ and $(b) \phi_{m}=0.3$. The parameter $z_{0}=D_{r} / S_{r}$ determines the non-dimensional length scale for order-unity changes in concentration. The horizontal dot-dash line corresponds to no diffusive remixing $\left(z_{0}=0\right)$ and the dashed line corresponds to no segregation $\left(z_{0}=\infty\right)$. For $z_{0} \in(0, \infty)$ graded particle-size distributions develop with increasingly sharper grading as $z_{0}$ decreases.

is the non-dimensional segregation-remixing length scale over which there are order unity changes in concentration. Note, that this is independent of the linear drag coefficient $c$ and is equal to the reciprocal of the Péclet number $P e=q H / D$. In time-dependent problems, the mean concentration $\phi_{m}$ is set by the initial conditions, since the boundaries insulate the problem from the exterior. The one-dimensional time-dependent segregation-remixing equation therefore has the property that the final steady state is uniquely determined by the initial conditions.

Steady-state solutions are illustrated in figure 3 for $z_{0}=0,0.02,0.1,0.5, \infty$ and $\phi_{m}=0.3,0.5$. All of them have ' $\mathrm{S}$ '-shaped profiles typical of the logistic equation. When there is no diffusive remixing $\left(z_{0}=0\right)$, the particles separate out to form two distinct inversely graded layers with all the large particles on top of all the fines and a sharp concentration jump at $z=\phi_{m}$. This is the case that Savage \& Lun (1988), Gray \& Thornton (2005), Thornton et al. (2006) and Gray, Shearer \& Thornton (2006) investigated theoretically and experimentally. At the opposite extreme, when there is no segregation $\left(z_{0} \longrightarrow \infty\right)$, the particles are uniformly distributed with concentration $\phi=\phi_{m}$ throughout the depth of the avalanche. For values of $z_{0}$ in the range $(0, \infty)$, there is a balance between the effects of particle-size segregation and diffusive remixing, and distributed inversely graded concentration profiles develop. For low values of $z_{0}$, there is a rapid transition from small to large particles with increasing height with states approaching the pure phases at the top and the bottom of the avalanche. For larger values of $z_{0}$, the grading appears linear with depth and the surface and basal values may be far from the pure states. The parameter dependence of $\phi(z)$ on $z_{0}$ is shown in figure 4 for $\phi_{m}=0.3,0.5$. These show that when $z_{0}=0$, a concentration shock lies at $z=\phi_{m}$ and that as $z_{0}$ is increased, the contours fan out, so that by $z_{0}=1$ there is a very weak inverse grading through the avalanche depth. When $\phi_{m}=0.5$, the contours of $\phi$ are straight lines in $\left(z_{0}, z\right)$ space. For a given contour level $\phi_{*}$ the equation of the line is

$$
z=\frac{1}{2}-z_{0} \ln \left(\frac{\phi_{*}}{1-\phi_{*}}\right)
$$




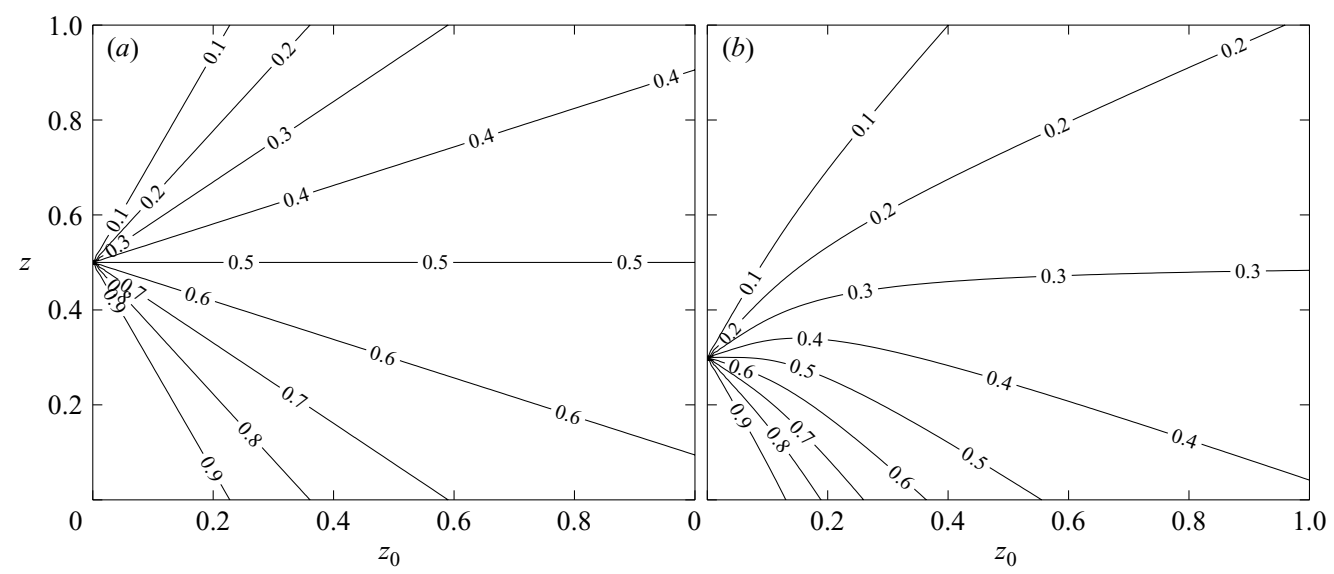

FIGURE 4. Contour plots showing the variation in the small-particle concentration $\phi$ as a function of depth $z$ and the segregation/diffusive remixing length scale $z_{0}=D_{r} / S_{r}$. (a) Average concentration $\phi_{m}=0.5 ;(b) \phi_{m}=0.3$.

In particular, the $\phi=0.5$ contour lies at a constant height $z=1 / 2$, so that the profiles all intersect at the same point in figure 3 . When $\phi_{m}$ is not equal to a half, the contours are no longer straight, but bend over with increasing $z_{0}$ as shown for $\phi_{m}=0.3$ in figure 4. As a result, the profiles do not neatly intersect at $(\phi, z)=\left(\phi_{m}, \phi_{m}\right)$ for $\phi_{m} \neq 1 / 2$, as shown in figure 3 .

\section{Comparison with particle dynamics simulations}

Accurately determining the volume fraction of small particles in a thin rapidly flowing granular avalanche presents many technical challenges and until non-invasive techniques are improved, samples of material must be collected from the deposit once the flow has come to rest. Savage \& Lun (1988) developed a splitter-plate arrangement that collected the outflow in a series of five bins. By varying the length of the chute they were able to build up a detailed spatial picture of the size distribution and the evolution towards a fully segregated state, although the binning procedure did not have enough resolution to determine the position of the concentration shocks that they observed through the sidewall. Dolgunin \& Ukolov (1995) binned samples from the cascading jet of material as it exited the chute. They claimed that their procedure could measure the outflow velocity, the particle concentration and the solids volume fraction. However, while the trajectory of a particle at a known height and exit velocity can be calculated using the laws of a free-falling body, the inverse problem does not have a unique solution. Additional assumptions must be made about the mapping from the chute exit to the bin, which are not described in the paper. Furthermore, their formula (17) for the exit velocity is dependent on time, which is incorrect, so there is an element of doubt about the data that they collected.

Until better experimental data become available, the best way of assessing the validity of the model is by using particle dynamics simulations which allow the local particle size distribution to be calculated. Kharkar et al. (1999) have performed a series of simulations using inelastic particles of different densities and sizes and at different solids volume fractions. The regime appropriate for size segregation in a dense granular avalanche is illustrated in their figure 19. In order to make a comparison to the segregation-remixing theory, their data must be transformed from number densities $n^{l}$ and $n^{s}$ for the large and small particles of diameters $d^{l}$ and $d^{s}$, 


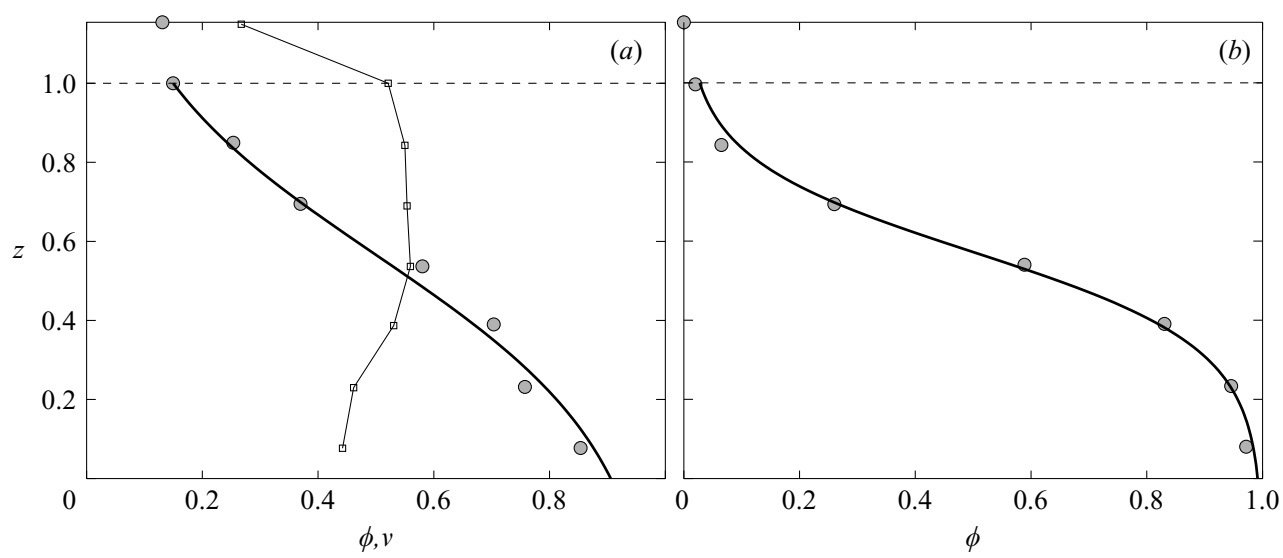

FiguRE 5. Comparison of the steady-state concentration with equilibrium particle dynamics simulations (Khakhar et al. 1999) for $(a)$ large and small particles and $(b)$ heavy and light particles. The size segregation simulations are performed with equal density inelastic particles with a size ratio $\sigma=0.9$ on a slope inclined at $\zeta=24^{\circ}$ and the data are indicated with grey circular markers. These are in good agreement with the segregation-remixing solution (thick solid line) with $\phi_{m}=0.55$ and $z_{0}=0.25$. The horizontal dashed line marks the approximate position of the avalanche/saltation layer interface, where the solids volume fraction $v$ drops well below 0.4 in the particle dynamics simulations as indicated by the square markers and the thin solid line. The density simulations $(b)$ are performed for equal size particles with a density ratio of 0.5 on the same slope and the density concentration is accurately modelled with parameters $\phi_{m}=0.57$ and $z_{0}=0.12$.

respectively, into the volume fraction $\phi$ of small particles. The relevant definitions are given by Savage \& Lun (1988) in equations (3.4), (3.5) and (4.7) who define the number density ratio and the particle diameter ratio as

$$
\eta=\frac{n^{s}}{n^{l}}, \quad \sigma=\frac{d^{s}}{d^{l}},
$$

and the corresponding small-particle concentration is then

$$
\phi=\frac{\eta \sigma^{3}}{1+\eta \sigma^{3}} .
$$

Figure 5(a) shows the transformed data from the particle dynamics simulations as grey circular markers at eight heights in the flow. A very good fit with the steady-state small-particle concentration is obtained by setting the value of the single parameter $z_{0}=0.25$ once the mean concentration is determined to be $\phi_{m}=0.55$. In particular, the fit is much better than that obtained by using kinetic theory with an effective granular temperature as a fitting parameter. The solids volume fraction $v$ in the particle dynamics simulations has been plotted on the same graph. This illustrates that while it is approximately constant throughout much of the depth $(v \simeq 0.45-0.55)$ consistent with the mono-disperse computations of Silbert et al. (2001) and Louge (2003), it drops significantly in the uppermost near-surface layer. This indicates the presence of what may be best described as a diffuse saltation layer at the free surface, where the dominant interactions are due to binary collisions rather than sliding or rolling contacts. There is therefore no reason to assume that the simple segregationremixing theory will apply here and we have indicated the approximate position of the transition with a horizontal dashed line. 
A similar segregation-remixing theory has been used before by Khakhar et al. (1997) to model density segregation in rotating drums and they obtained good agreement with experiments, Monte Carlo methods and particle dynamics simulations. Further evidence that the proposed segregation-remixing theory is also useful for modelling density segregation in granular avalanches is given by comparing it to the particle dynamics simulations of Khakhar et al. (1999). Transforming the data shown in their figure 9 for inelastic particles with a density ratio of 0.5 into a volume fraction $\phi$ of dense particles, a good fit to the segregation-remixing theory is obtained with parameters $\phi_{m}=0.57$ and $z_{0}=0.12$. This predicts that dense particles concentrate themselves near the base of the flow and the light particles rise to the surface as anticipated. Once again, the agreement is better than that obtained with kinetic theory with an effective temperature that is used as a fitting parameter, which provides further evidence that this structure is useful for modelling segregation in dense granular avalanches.

At present, the theory does not provide explicit formulae or empirical relations for the magnitude of the segregation and remixing coefficients and their dependence on the particle-size, particle density, local shear rate, slope inclination, degree of agitation or solids volume fraction. In particular, it is unclear which effect will dominate when there are both size and density differences between the particles. However, particle dynamics appears to provide a useful avenue for answering these questions and calibrating the model.

\section{Time-dependent solutions for uniform flows}

Time-dependent exact solutions to the segregation-remixing equation (2.20) have been constructed when the diffusion coefficient $D_{r}$ is equal to zero, and the system reduces to a quasi-linear equation, which can be solved by the method of characteristics (Gray \& Thornton 2005, Thornton et al. 2006, Gray et al. 2006). It is of interest to construct solutions to the full parabolic equation (2.20) when the effects of diffusion are included.

\subsection{Uniform flow}

To construct time-dependent solutions for segregation and remixing, the flow is assumed to be laterally uniform and there are no lateral gradients in the smallparticle concentration

$$
\begin{gathered}
u=u(z), \quad v=v(z), \quad w=0, \quad O<z<1, \\
\partial \phi / \partial x=0, \quad \partial \phi / \partial y=0 .
\end{gathered}
$$

These conditions imply that the segregation-remixing equation (2.20) reduces to

$$
\frac{\partial \phi}{\partial t}-S_{r} \frac{\partial}{\partial z}(\phi(1-\phi))=D_{r} \frac{\partial^{2} \phi}{\partial z^{2}},
$$

which is independent of $u(z)$ and $v(z)$. This is important, because it implies that the results hold for arbitrary lateral velocity profiles, from plug flow, which is usually assumed in most avalanche models, to simple shear (Savage \& Lun 1988) and nonlinear profiles (e.g. Silbert et al. 2001). Equation (5.3) must be solved subject to an initial concentration profile $\phi_{0}(z)$ and the no-flux boundary condition (2.24) at the surface and base of the avalanche

$$
t=0: \quad \phi=\phi_{0}(z)
$$




$$
z=0,1: \quad S_{r} \phi(1-\phi)+D_{r} \frac{\partial \phi}{\partial z}=0 .
$$

The reduced segregation-remixing equation (5.3) is a nonlinear parabolic PDE that is subject to nonlinear boundary conditions (5.5) at its upper and lower boundaries.

\subsection{Transformation and general solution of the equations}

The method of solution relies on a sequence of transformations of the segregationremixing equation (5.3) and the initial and boundary conditions (5.4)-(5.5) to obtain a linear system that can be solved by standard methods. The first transformation scales the time and space axes and introduces a concentration mapping

$$
t=\left(\frac{D_{r}}{S_{r}^{2}}\right) \tau, \quad z=\left(\frac{D_{r}}{S_{r}}\right) \zeta, \quad \phi=\frac{1}{2}(1+\Psi),
$$

to obtain a convenient parameter independent form of the equations

$$
\begin{array}{cc}
\frac{\partial \Psi}{\partial \tau}+\Psi \frac{\partial \Psi}{\partial \zeta}=\frac{\partial^{2} \Psi}{\partial \zeta^{2}} \quad\left(0<\zeta<\zeta_{0}\right), \\
\tau=0: \quad & \Psi=\Psi_{0}=2 \phi_{0}-1, \\
\zeta=0, \zeta_{0}: & \frac{1}{2}\left(1-\Psi^{2}\right)+\frac{\partial \Psi}{\partial \zeta}=0,
\end{array}
$$

where $\zeta_{0}=1 / z_{0}$ is the number of non-dimensional segregation-remixing lengths $z_{0}$ that fit into an avalanche of unit height. The concentration mapping has transformed the segregation-remixing equation (5.3) into the Burgers equation (5.7), whose properties are well known (e.g. Whitham 1974; Billingham \& King 2000; Cantwell 2002). In particular, solutions to (5.7) may be constructed by using the Cole-Hopf transformation (Hopf 1950; Cole 1951)

$$
\Psi=-\frac{2}{\chi} \frac{\partial \chi}{\partial \zeta}
$$

to map the Burgers equation into the heat equation

$$
\frac{\partial \chi}{\partial \tau}=\frac{\partial^{2} \chi}{\partial \zeta^{2}} \quad\left(0<\zeta<\zeta_{0}\right)
$$

and the initial and boundary conditions to

$$
\begin{aligned}
\tau=0: & \chi=\chi_{0}(\zeta)=\exp \left(\int_{0}^{\zeta}-\frac{1}{2} \Psi_{0}\left(\zeta^{\prime}\right) \mathrm{d} \zeta^{\prime}\right), \\
\zeta=0, \zeta_{0}: & \frac{\partial^{2} \chi}{\partial \zeta^{2}}=\frac{1}{4} \chi .
\end{aligned}
$$

The transformed problem (5.11) is now linear with linear boundary conditions (5.13). In order to further simplify the boundary conditions (5.13), a solution of the form

$$
\chi=\exp (\alpha \tau) \omega(\zeta, \tau)
$$

is sought, which implies that

$$
\alpha \omega+\frac{\partial \omega}{\partial \tau}=\frac{\partial^{2} \omega}{\partial \zeta^{2}}
$$




$$
\begin{aligned}
\tau=0: & \omega=\chi_{0}(\zeta), \\
\zeta=0, \zeta_{0}: & \frac{\partial^{2} \omega}{\partial \zeta^{2}}=\frac{1}{4} \omega .
\end{aligned}
$$

At steady state, $\partial^{2} \omega_{s} / \partial \zeta^{2}=\alpha \omega_{s}$ and hence the boundary conditions reduce to

$$
\zeta=0, \zeta_{0}: \quad\left(\alpha-\frac{1}{4}\right) \omega_{s}=0
$$

Since the steady-state solution $\omega_{s}(\zeta)$ is not equal to zero at the boundaries, equation (5.18) implies that both boundary conditions (5.17) are satisfied provided $\alpha=1 / 4$. Putting $\alpha=1 / 4$ in the transformation (5.14) implies that the term $\partial^{2} \omega / \partial \zeta^{2}-\omega / 4$ is common to equation (5.15) and the boundary conditions (5.17), which necessarily implies that $\partial \omega / \partial \tau=0$ at $\zeta=0, \zeta_{0}$. The boundary values therefore remain constant and equal to their initial values throughout the evolution of the transformed problem. Hence, the initial condition (5.16) implies that the boundary conditions (5.17) reduce to

$$
\begin{array}{ll}
\zeta=0: & \omega=1, \\
\zeta=\zeta_{0}: & \omega=\chi_{0}\left(\zeta_{0}\right)=\exp \left(\int_{0}^{\zeta_{0}}-\frac{1}{2} \Psi_{0}\left(\zeta^{\prime}\right) \mathrm{d} \zeta^{\prime}\right) .
\end{array}
$$

Considerable simplification of the problem can be achieved by breaking the solution down into a steady-state and time varying part, i.e.

$$
\omega(\zeta, \tau)=\omega_{s}(\zeta)+\omega_{\tau}(\zeta, \tau)
$$

where the steady-state solution is

$$
\omega_{s}=\chi_{0}\left(\zeta_{0}\right) \frac{\sinh (\zeta / 2)}{\sinh \left(\zeta_{0} / 2\right)}-\frac{\sinh \left(\left(\zeta-\zeta_{0}\right) / 2\right)}{\sinh \left(\zeta_{0} / 2\right)} .
$$

Reversing the transformations (5.6), (5.10) and (5.14) implies that the corresponding steady-state concentration $\phi_{s}(z)$ is equal to

$$
\phi_{s}=\frac{1}{2}\left[1-\frac{\chi_{0}\left(\zeta_{0}\right) \cosh \left(z /\left(2 z_{0}\right)\right)-\cosh \left(\left(z-z_{0}\right) /\left(2 z_{0}\right)\right)}{\chi_{0}\left(\zeta_{0}\right) \sinh \left(z /\left(2 z_{0}\right)\right)-\sinh \left(\left(z-z_{0}\right) /\left(2 z_{0}\right)\right)}\right],
$$

where $\chi_{0}\left(\zeta_{0}\right)=\exp \left\{-\left(2 \phi_{m}-1\right) /\left(2 z_{0}\right)\right\}$. Using the definitions of the hyperbolic functions, it is easy to show that $(5.23)$ is precisely the same as the steady-state solution (3.4) investigated in $\S 3$. The remaining time-dependent part of the solution is of standard form

$$
\begin{array}{cl}
\frac{\partial \omega_{\tau}}{\partial \tau}=\frac{\partial^{2} \omega_{\tau}}{\partial \zeta^{2}}-\frac{1}{4} \omega_{\tau} \\
\tau=0: \quad \omega_{\tau}=\chi_{0}(\zeta)-\omega_{s}(\zeta), \\
\zeta=0, \zeta_{0}: \quad \omega_{\tau}=0 .
\end{array}
$$

Using the separation of variables $\omega_{\tau}=T(\tau) Z(\zeta)$ gives

$$
Z^{\prime \prime}+\left(\lambda^{2}-1 / 4\right) Z=0, \quad \zeta=0, \zeta_{0}: Z=0,
$$

and

$$
\dot{T}+\lambda^{2} T=0
$$


where the prime denotes $\mathrm{d} / \mathrm{d} z$ and the over dot $\mathrm{d} / \mathrm{d} t$. The only non-trivial solutions of the eigenvalue problem (5.27) are

$$
\lambda^{2}=\left(\frac{n \pi}{\zeta_{0}}\right)^{2}+\frac{1}{4}, \quad Z(\zeta)=\sin \left(\frac{n \pi \zeta}{\zeta_{0}}\right)
$$

where $n$ is a strictly positive integer. The corresponding solutions for $T$ are

$$
T=\exp \left(-\left(\frac{n^{2} \pi^{2}}{\zeta_{0}^{2}}+\frac{1}{4}\right) \tau\right)
$$

and the principle of linear superposition implies that the general solution is

$$
\omega_{\tau}=\sum_{n=1}^{\infty} A_{n} \exp \left(-\left(\frac{n^{2} \pi^{2}}{\zeta_{0}^{2}}+\frac{1}{4}\right) \tau\right) \sin \left(\frac{n \pi \zeta}{\zeta_{0}}\right),
$$

where the coefficients $A_{n}$ are determined from the initial conditions. At $\tau=0$, equation (5.31) reduces to a Fourier sine series for $\omega_{\tau}(\zeta, 0)$ and the Fourier coefficients $A_{n}$ are therefore given by

$$
A_{n}=\frac{2}{\zeta_{0}} \int_{0}^{\zeta_{0}} \omega_{\tau}(\zeta, 0) \sin \left(\frac{n \pi \zeta}{\zeta_{0}}\right) \mathrm{d} \zeta .
$$

From the initial condition (5.25), $A_{n}$ can be decomposed into the difference of two integrals, $A_{n}=B_{n}-C_{n}$, where

$$
B_{n}=\frac{2}{\zeta_{0}} \int_{0}^{\zeta_{0}} \chi_{0}(\zeta) \sin \left(\frac{n \pi \zeta}{\zeta_{0}}\right) \mathrm{d} \zeta, \quad C_{n}=\frac{2}{\zeta_{0}} \int_{0}^{\zeta_{0}} \omega_{s}(\zeta) \sin \left(\frac{n \pi \zeta}{\zeta_{0}}\right) \mathrm{d} \zeta .
$$

The first integral, $B_{n}$, is dependent on the functional form of the transformed initial conditions, $\chi_{0}(\zeta)$, defined in (5.12). The second integral $C_{n}$ is dependent on the steadystate solution $\omega_{s}$, given by (5.22), and can be integrated for general initial conditions since the factor $\chi_{0}\left(\zeta_{0}\right)$, defined in (5.20), is constant, i.e.

$$
C_{n}=\frac{8 n \pi}{\zeta_{0}^{2}+4 n^{2} \pi^{2}}\left(1-(-1)^{n} \chi_{0}\left(\zeta_{0}\right)\right)
$$

Putting the transformations (5.6), (5.10), (5.14) and (5.21) together, it follows that the general solution of the segregation-remixing equation (5.3), for arbitrary initial concentration profiles $\phi_{0}(z)$ and the no-flux boundary conditions (5.5) at the surface and base of the avalanche, is

$$
\phi=\left.\frac{1}{2}\left(1-\frac{2}{\omega} \frac{\partial \omega}{\partial \zeta}\right)\right|_{\zeta=z / z_{0}, \tau=S_{r} t / z_{0}}
$$

where $\omega$ is decomposed into a steady and time-varying part

$$
\omega=\omega_{s}+\omega_{\tau} .
$$

These functions and their derivatives are given by

$$
\begin{aligned}
\omega_{s} & =\chi_{0}\left(\zeta_{0}\right) \frac{\sinh (\zeta / 2)}{\sinh \left(\zeta_{0} / 2\right)}-\frac{\sinh \left(\left(\zeta-\zeta_{0}\right) / 2\right)}{\sinh \left(\zeta_{0} / 2\right)}, \\
\frac{\partial \omega_{s}}{\partial \zeta} & =\frac{1}{2} \chi_{0}\left(\zeta_{0}\right) \frac{\cosh (\zeta / 2)}{\sinh \left(\zeta_{0} / 2\right)}-\frac{1}{2} \frac{\cosh \left(\left(\zeta-\zeta_{0}\right) / 2\right)}{\sinh \left(\zeta_{0} / 2\right)},
\end{aligned}
$$




$$
\begin{gathered}
\omega_{\tau}=\sum_{n=1}^{\infty} A_{n} \exp \left(-\left(\frac{n^{2} \pi^{2}}{\zeta_{0}^{2}}+\frac{1}{4}\right) \tau\right) \sin \left(\frac{n \pi \zeta}{\zeta_{0}}\right), \\
\frac{\partial \omega_{\tau}}{\partial \zeta}=\sum_{n=1}^{\infty} \frac{n \pi}{\zeta_{0}} A_{n} \exp \left(-\left(\frac{n^{2} \pi^{2}}{\zeta_{0}^{2}}+\frac{1}{4}\right) \tau\right) \cos \left(\frac{n \pi \zeta}{\zeta_{0}}\right),
\end{gathered}
$$

where the coefficients $\chi_{0}\left(\zeta_{0}\right)$ and $A_{n}$ must be determined from equations (5.20) and (5.32), respectively, once the initial conditions are specified. Some simple cases are investigated in $\S \S 5.3-5.5$.

\subsection{Homogeneous initial conditions}

Consider the problem in which the avalanche is laterally uniform and is assumed to have a homogeneous initial distribution of small particles with depth

$$
\phi_{0}=\phi_{m}, \quad 0<z<1,
$$

where $\phi_{m}$ is the mean concentration. To construct the solution the constant $\chi_{0}\left(\zeta_{0}\right)$ and the Fourier coefficients $A_{n}$ in the general solution (5.35)-(5.40) must be determined. The concentration mapping (5.8) implies that

$$
\Psi_{0}=\Psi_{m}=2 \phi_{m}-1, \quad 0<\zeta<\zeta_{0} .
$$

Since $\Psi_{m}$ is constant, the integrals (5.12) and (5.20) imply that

$$
\chi_{0}(\zeta)=\exp \left(-\Psi_{m} \zeta / 2\right), \quad \chi_{0}\left(\zeta_{0}\right)=\exp \left(-\Psi_{m} \zeta_{0} / 2\right),
$$

which determines the unknown function in the integral (5.33) for $B_{n}$ and the constant in the expression (5.34) for $C_{n}$. Using the standard result

$$
\int \exp (\alpha \zeta) \sin (\beta \zeta) \mathrm{d} \zeta=\frac{\exp (\alpha \zeta)}{\alpha^{2}+\beta^{2}}(\alpha \sin (\beta \zeta)-\beta \cos (\beta \zeta)),
$$

it is easy to show that the integral

$$
B_{n}=\frac{8 n \pi}{\Psi_{m}^{2} \zeta_{0}^{2}+4 n^{2} \pi^{2}}\left(1-(-1)^{n} \exp \left(-\Psi_{m} \zeta_{0} / 2\right)\right),
$$

and hence that the coefficients in the Fourier sine series are

$$
A_{n}=\frac{8 n \pi \zeta_{0}^{2}\left(1-\Psi_{m}^{2}\right)}{\left(\Psi_{m}^{2} \zeta_{0}^{2}+4 n^{2} \pi^{2}\right)\left(\zeta_{0}^{2}+4 n^{2} \pi^{2}\right)}\left(1-(-1)^{n} \exp \left(-\Psi_{m} \zeta_{0} / 2\right)\right) .
$$

The solution is therefore given by equations (5.35)-(5.40) with $\chi_{0}\left(\zeta_{0}\right)$ defined in (5.43) and the Fourier coefficients $A_{n}$ given by (5.46). The results for varying $D_{r}$ and $\phi_{m}=0.55$ and 0.35 are illustrated in figure 6 . The upper middle panels show the case for $D_{r}=0.02$ and $S_{r}=1.0$, which implies that the segregation-remixing length scale $z_{0}$, defined in (3.5), is equal to 0.02 . For short time, the concentration stays close to its initial value $\phi_{m}$ throughout most of the depth, and the small particles percolate downwards levering the large grains upwards. At the free surface, the boundary condition (5.5) ensures that no more small particles are fed in and the large particles start to accumulate. As opposed to the solutions of Gray \& Thornton (2005), the large particles do not separate out into a pure phase across a concentration shock. Instead there is a continuous sharp transition over the segregation-remixing length scale $z_{0}$. After $t=0.4$, a region approaching a pure phase of large particles begins to develop at the surface. A similar smooth transition to a pure phase of small particles develops at the bottom. With increasing time-both the pure phase regions grow in size 

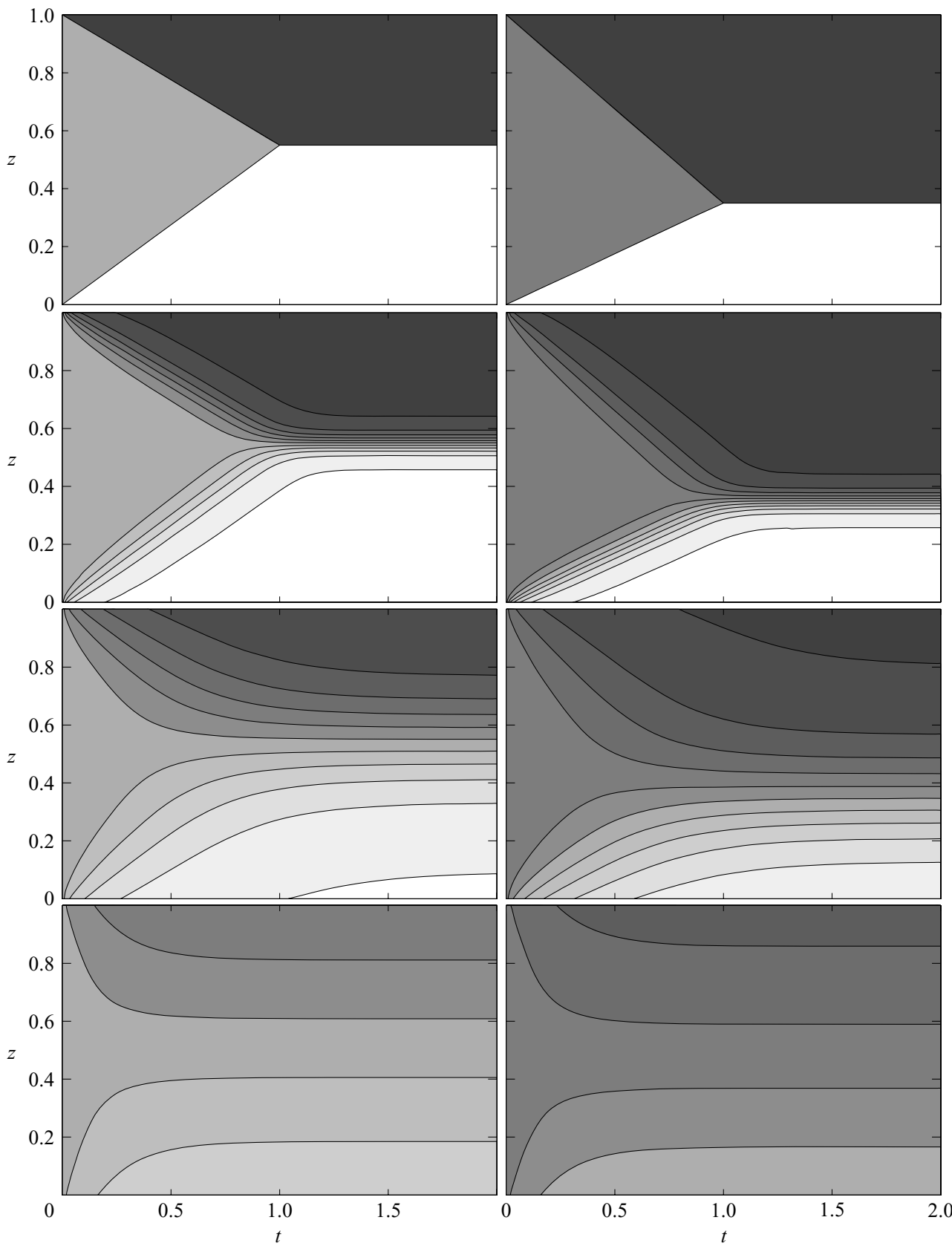

Large

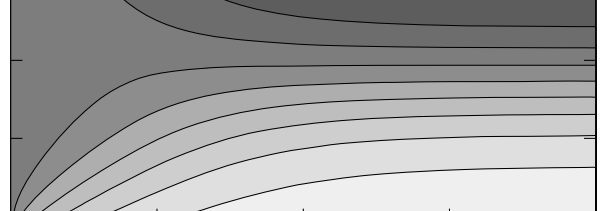


and by $t=1.0$ the large central region at the initial concentration $\phi_{m}$ is annihilated, and instead there is rapid transition from an almost pure phase of large particles to almost pure fines. By $t=2$, the solution is very close to the steady states discussed in $\S 3$.

It is interesting to contrast the low diffusion limit with the shock solutions derived by Gray \& Thornton (2005) for $D_{r}=0$. As illustrated in the top two panels of figure 6 , these consist of three linearly time-dependent shocks

$$
\left.\begin{array}{ll}
z_{1}=S_{r} \phi_{m} t, & 0 \leqslant t \leqslant 1 / S_{r}, \\
z_{2}=1-S_{r}\left(1-\phi_{m}\right) t, & 0 \leqslant t \leqslant 1 / S_{r} \\
z_{3}=\phi_{m}, & t>1 / S_{r},
\end{array}\right\}
$$

that separate regions of constant concentration from one another

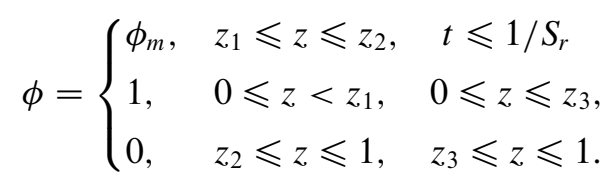

Qualitatively the solutions look very similar to those when the segregation-remixing length scale $z_{0}=0.02$, except that the rapid smooth transitions are replaced by shocks. Indeed, the time scale for the annihilation of the initial concentration region at $t=1 / S_{r}$ is almost identical to the low remixing case.

For larger segregation-remixing lengths $z_{0}$, order-unity changes in concentration occur over longer length and time scales by virtue of the scalings (5.6) and the contours for $D_{r}=0.1$ and 0.5 , shown in the lower middle and bottom panels of figure 6 , are much more spread out than when $D_{r}=0.02$. An important feature of these solutions is that the initial condition (5.41) is incompatible with the boundary conditions (5.5) at $z=0,1$, since the initial conditions are uniform with depth and the boundary conditions necessarily imply that there is a finite gradient at the boundary. The net result is that as the large particles gather at the free surface, and the fines percolate to the base, there is a very rapid local adjustment as the solution satisfies the no normal flux condition (5.5). This adjustment produces large gradients that are then rapidly diffused into the interior, with the strength increasing with increasing $z_{0}$. As a result, the homogeneous triangular region in the shock solutions (5.48) is rapidly annihilated when $D_{r}=0.5$. Note that the incompatibility of the boundary conditions is a stern test of numerical methods to solve the segregation-remixing equation. All of the solutions tend towards the steady-state solutions described in $\S 3$ by $t=2$.

\subsection{Reverse graded initial conditions}

Ensuring that the initial concentration distribution is laterally uniform and homogeneous with depth is difficult to do experimentally. It is, however, much easier to create layered initial distributions in the laboratory, and our second problem is motivated by this. Let us suppose that initially a pure layer of large grains overlies a pure region of fines with a sharp concentration jump at $z=z_{r}$, i.e.

$$
\phi_{0}= \begin{cases}0, & z_{r}<z<1 \\ 1, & 0<z<z_{r}\end{cases}
$$

In the absence of diffusive remixing, the shock condition (2.22) reduces to

$$
\partial z / \partial t=S_{r}\left(\phi^{+}+\phi^{-}-1\right)
$$


(see also Gray \& Thornton 2005), where the concentration above the shock $\phi^{+}=0$ and the concentration below the shock $\phi^{-}=1$. This has the trivial solution

$$
z=z_{r}, \quad t>0 \text {, }
$$

which implies that the initial condition is a stable solution of pure segregation equations. When there is diffusive remixing, the sharp interface is diffused. This time, (5.8) implies that

$$
\Psi_{0}=\left\{\begin{aligned}
-1, & \zeta_{r}<\zeta<\zeta_{0} \\
1, & 0<\zeta<\zeta_{r}
\end{aligned}\right.
$$

where $\zeta_{r}=z_{r} / z_{0}$. Integrating over the appropriate ranges, (5.12) implies that

$$
\chi_{0}(\zeta)= \begin{cases}\exp \left(\zeta / 2-\zeta_{r}\right), & \zeta_{r}<\zeta<\zeta_{0} \\ \exp (-\zeta / 2), & 0<\zeta<\zeta_{r}\end{cases}
$$

and hence the constant (5.20) is simply

$$
\chi_{0}\left(\zeta_{0}\right)=\exp \left(\zeta_{0} / 2-\zeta_{r}\right)
$$

Equation (5.53) implies that the integral (5.33) for $B_{n}$ must be split into two parts

$$
B_{n}=\frac{2}{\zeta_{0}} \int_{0}^{\zeta_{r}} \exp (-\zeta / 2) \sin \left(\frac{n \pi \zeta}{\zeta_{0}}\right) \mathrm{d} \zeta+\frac{2}{\zeta_{0}} \int_{\zeta_{r}}^{\zeta_{0}} \exp \left(\zeta / 2-\zeta_{r}\right) \sin \left(\frac{n \pi \zeta}{\zeta_{0}}\right) \mathrm{d} \zeta
$$

which can easily be evaluated using (5.44) to show that

$$
B_{n}=\frac{-8 \zeta_{0}}{\zeta_{0}^{2}+4 n^{2} \pi^{2}}\left\{\exp \left(-\zeta_{r} / 2\right) \sin \left(\frac{n \pi \zeta_{r}}{\zeta_{0}}\right)-\frac{n \pi}{\zeta_{0}}\left(1-(-1)^{n} \exp \left(\zeta_{0} / 2-\zeta_{r}\right)\right)\right\} \text {. }
$$

The second term in the curly brackets cancels out with $C_{n}$, so that the Fourier coefficients are given by

$$
A_{n}=\frac{-8 \zeta_{0}}{\zeta_{0}^{2}+4 n^{2} \pi^{2}} \exp \left(-\zeta_{r} / 2\right) \sin \left(\frac{n \pi \zeta_{r}}{\zeta_{0}}\right)
$$

The full solution is therefore given by equations $(5.35)-(5.40)$ with $\chi_{0}\left(\zeta_{0}\right)$ given by (5.54) and the Fourier coefficients by (5.57). The shock solution for $D_{r}=0$ and the solutions for three non-zero values of segregation-remixing length $z_{0}$ are illustrated in figure 7. The upper middle panels show that for $z_{0}=0.02$, the sharp shock interface is diffused over a relatively short smooth transition and the solution rapidly attains its steady state, consistent with the scalings (5.6). As $z_{0}$ is increased to 0.1 , the initial concentration shock is diffused over a much wider range and takes significantly longer to reach steady state. The initial discontinuity at $z=z_{r}$ is rapidly smoothed away by diffusive-remixing and the concentration contours therefore emerge from $\left(z_{r}, 0\right)$ with an infinite gradient. When $z_{0}=0.5$, the initial response becomes even stronger, which, once again, provides a stern test for numerical methods. The solutions tend towards the steady-state solutions illustrated in figures 3 and 4 with increasing time.

\subsection{Normally graded initial conditions}

If the initial configuration of $\S 5.4$ is reversed so that a pure region of fines now lies above the coarse grains with a sharp concentration jump at $z=z_{r}$

$$
\phi_{0}= \begin{cases}1, & z_{r}<z<1 \\ 0, & 0<z<z_{r}\end{cases}
$$




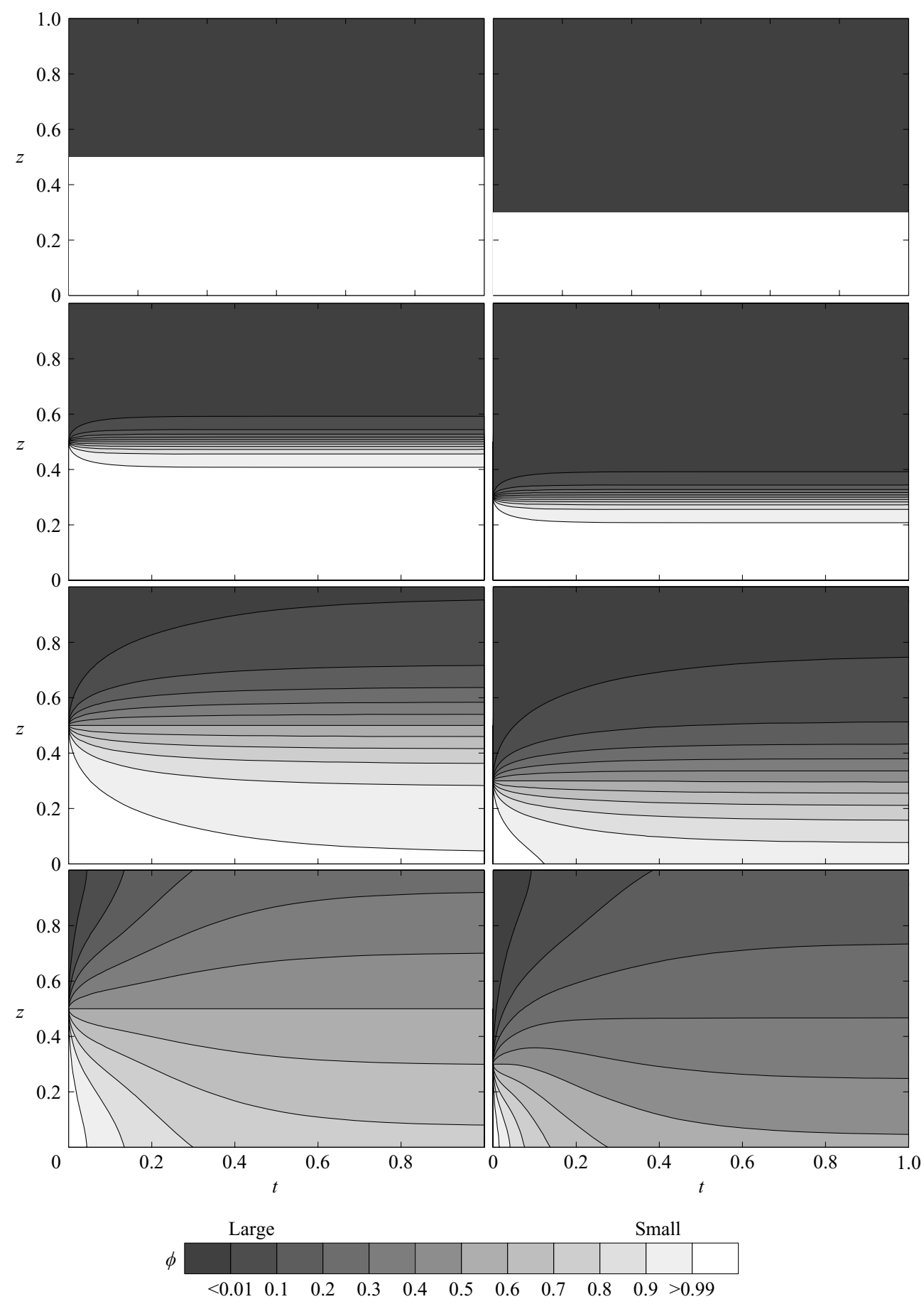

FIGURE 7. Contour plot showing the evolution of the small-particle concentration $\phi$ as a function of the avalanche depth $z$ and time $t$. The concentration is laterally, uniform throughout the flow field and the solution holds for arbitrary downslope and cross-slope velocities. Initially, the flow is reverse graded with a sharp jump in concentration at $z_{r}=0.5$ in the left-hand panels and $z_{r}=0.3$ in the right-hand panels. The segregation number $S_{r}=1$ in all of the panels and the diffusive remixing coefficient $D_{r}$ takes the values $0,0.02,0.1$ and 0.5 from top to bottom, respectively. Lighter greys correspond to larger concentrations of small particles. 
then the grains are unstably stratified and the solution must readjust into a stable reverse-graded configuration. To construct the solution, the concentration mapping (5.8) transforms the initial conditions for Burgers equation to

$$
\Psi_{0}=\left\{\begin{aligned}
1, & \zeta_{r}<\zeta<\zeta_{0} \\
-1, & 0<\zeta<\zeta_{r}
\end{aligned}\right.
$$

the second mapping (5.10) therefore implies that

$$
\chi_{0}(\zeta)= \begin{cases}\exp \left(\zeta_{r}-\zeta / 2\right), & \zeta_{r}<\zeta<\zeta_{0} \\ \exp (\zeta / 2), & 0<\zeta<\zeta_{r}\end{cases}
$$

and hence

$$
\chi_{0}\left(\zeta_{0}\right)=\exp \left(\zeta_{r}-\zeta_{0} / 2\right)
$$

This determines the steady-state part of the solution (5.37). To construct the timedependent part, the integral $B_{n}$ in (5.33) must be evaluated. The function (5.60) implies that the integral can be evaluated by splitting it into two parts, as in (5.55), to give

$$
B_{n}=\frac{8 \zeta_{0}}{\zeta_{0}^{2}+4 n^{2} \pi^{2}}\left\{\exp \left(\zeta_{r} / 2\right) \sin \left(\frac{n \pi \zeta_{r}}{\zeta_{0}}\right)+\frac{n \pi}{\zeta_{0}}\left(1-(-1)^{n} \exp \left(\zeta_{r}-\zeta_{0} / 2\right)\right)\right\} .
$$

The second term in the curly brackets cancels out with $C_{n}$ and the Fourier coefficients in the time-dependent solution (5.39) reduce to

$$
A_{n}=\frac{8 \zeta_{0}}{\zeta_{0}^{2}+4 n^{2} \pi^{2}} \exp \left(\zeta_{r} / 2\right) \sin \left(\frac{n \pi \zeta_{r}}{\zeta_{0}}\right)
$$

The solution is therefore given by (5.35)-(5.40) with the coefficients given by (5.61) and (5.63) and are illustrated in figure 8 together with the no-diffusion limit derived by Thornton et al. (2006). This consists of an expansion fan, three shocks and four pure regions, where the small-particle concentration

$$
\phi=\left\{\begin{array}{cl}
1, & z_{r}+S_{r} t \leqslant z \leqslant 1, \quad 0 \leqslant z \leqslant z_{1} \cup z_{3}, \\
\frac{1}{2}\left(1+\frac{z-z_{r}}{S_{r} t}\right), & \left|z-z_{r}\right|<S_{r} t, \quad z_{1}<z<z_{2}, \\
0, & 0 \leqslant z \leqslant z_{r}-S_{r} t, \quad \& \quad z_{2} \cup z_{3} \leqslant z \leqslant 1,
\end{array}\right.
$$

the shocks

$$
\left.\begin{array}{ll}
z_{1}=z_{r}+S_{r} t-2 \sqrt{S_{r} z_{r} t}, & 0 \leqslant t \leqslant t_{p}, \\
z_{2}=z_{r}-S_{r} t+2 \sqrt{S_{r}\left(1-z_{r}\right) t}, & 0 \leqslant t \leqslant t_{p} \\
z_{3}=1-z_{r}, & t>t_{p},
\end{array}\right\}
$$

and the triple-point

$$
t_{p}=\frac{1}{S_{r}}\left(\sqrt{z_{r}}+\sqrt{1-z_{r}}\right)^{2} .
$$

The large particles start at the bottom and move downslope until they feel the first small particles percolating down through the matrix and they then begin to be levered upwards. The large particles rise though the expansion fan until they reach the top 

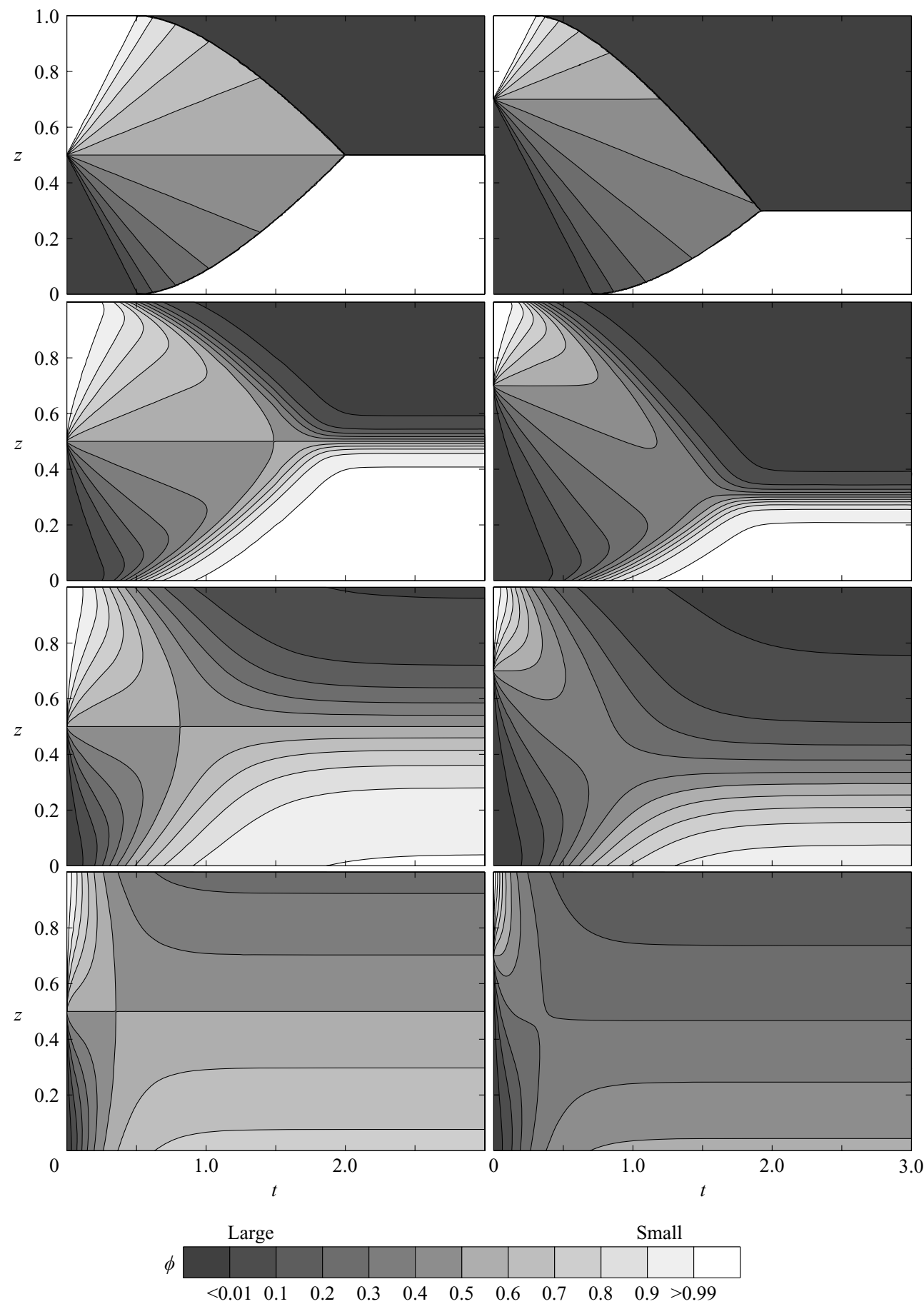

FIGURE 8. Contour plot showing the evolution of the small-particle concentration $\phi$ as a function of the avalanche depth $z$ and time $t$. The concentration is laterally uniform throughout the flow field and the solution holds for arbitrary downslope and cross-slope velocities. Initially, the flow is normally graded with a sharp jump in concentration at $z_{r}=0.5$ in the left-hand panels and $z_{r}=0.7$ in the right-hand panels. The segregation number $S_{r}=1$ in all of the panels and the diffusive remixing coefficient $D_{r}$ takes the values $0,0.02,0.1$ and 0.5 from top to bottom, respectively. Lighter greys correspond to larger concentrations of small particles. 
shock, $z_{2}$, where they separate out into a pure phase and move straight downstream again. The small particle paths are similar, except that they start at the top, move down through the expansion region and separate out into a pure phase at the bottom. After time $t_{p}$, the flow has reached a steady state with all the large particles on top of all the fines.

When $z_{0}=0.02$, the solution looks very similar to the no-diffusion limit except that the expansion fan is slightly wider and the shocks are replaced by rapid smooth transitions. The fan spreads out more quickly because diffusive remixing very rapidly smoothes out the initial discontinuity, which helps the large and small particles to reach the surface and base of the flow, thus aiding segregation. This effect becomes stronger at larger values of the segregation-remixing length $z_{0}=0.1$ and 0.5 , as illustrated in the lower-middle and bottom panels of figure 8. For larger $z_{0}$, the final grading of the particles is not as strong and the large and small particles therefore have less far to travel to reach their steady-state positions. The combined effects of the smoothing of the discontinuity and the particles having less far to travel to reach their final heights means that steady state is attained faster for larger $z_{0}$ when $S_{r}=1$.

\section{Time-dependent solutions for plug-flow in a semi-infinite chute}

In this section, some simplified time-dependent solutions for segregation and remixing in a semi-infinite chute are constructed. The method is based on the observation of Gray \& Thornton (2005) that in plug-flow, each moving column of material is uncoupled from the adjacent columns. The segregation-remixing equation (2.20) for plug-flow is

$$
\frac{\partial \phi}{\partial t}+u_{0} \frac{\partial \phi}{\partial x}-S_{r} \frac{\partial}{\partial z}(\phi(1-\phi))=D_{r} \frac{\partial^{2} \phi}{\partial z^{2}},
$$

where the transport velocity $u_{0}$ can, without loss of generality, be assumed to be unity by virtue of the scalings in (2.19). In a frame of reference moving downstream with speed $u_{0}$, given by the transformation of variables

$$
\hat{t}=t-t_{s}, \quad \xi=x-u_{0} t, \quad \hat{z}=z,
$$

the segregation-remixing equation (6.1) reduces to

$$
\frac{\partial \phi}{\partial \hat{t}}-S_{r} \frac{\partial}{\partial \hat{z}}(\phi(1-\phi))=D_{r} \frac{\partial^{2} \phi}{\partial \hat{z}^{2}} .
$$

Since this is independent of the column coordinate $\xi$, the segregation-remixing equation in a fixed moving column of material is completely independent of the segregation taking place in the adjacent columns. Equation (6.3) has exactly the same form as (5.3), which was investigated in section $\S 5$. The crucial difference is that $\hat{t}$ replaces $t$ and the transformation (6.2) therefore introduces a time-shift $t_{s}$ into the general solution given by (5.35)-(5.40). Temporally and spatially varying solutions can therefore be constructed by considering a series of adjacent material columns, all moving downslope at speed $u_{0}$, in which the time-shift $t_{s}(\xi)$, the coefficient $\chi_{0}\left(\zeta_{0}, \xi\right)$ and the Fourier coefficients $A_{n}(\xi)$ are functions of the column coordinate $\xi$.

Consider a steady uniform plug-flow on a semi-infinite chute $(x>0)$ that is supplied with granular material with a prescribed concentration distribution from a hopper at $x=0$. The coordinate $\xi$ is used to label each column of material uniquely by using 
either its initial position

$$
\xi=x \quad \text { at } \quad t=0
$$

or its effective position $x<0$, assuming that it reaches the mouth of the hopper at time $t_{\text {exit }}=-x / u_{0}$. It follows from (6.2) that at time $t$, column $\xi$ lies at

$$
x=\xi+u_{0} t .
$$

Columns with positive $\xi$ are therefore initially within the avalanche, whilst columns with negative $\xi$ first enter the avalanche, at $x=0$, at time $t_{\text {exit }}=-\xi / u_{0}$. The transition point, $\xi=0$, between the columns initially within the avalanche and those that subsequently enter it, is transported downstream with constant speed $u_{0}$ and has position $x_{\text {trans }}=u_{0} t$.

\subsection{Steady-state solutions in a semi-infinite chute}

Consider the case when the time-shift parameter is equal to the exit time for all columns irrespective of whether they were initially in the chute or entered it at a later time, i.e.

$$
t_{s}=-\xi / u_{0}, \quad \forall \xi
$$

The transformation (6.2) and equation (6.5) therefore imply

$$
\hat{t}=t+\xi / u_{0}=x / u_{0}
$$

which shows that the time-dependent evolution in a column of material moving downstream at speed $u_{0}$ is equivalent to spatial dependence in a fixed frame of reference. If, in addition, there is no variation in the column parameters $\chi_{0}\left(\zeta_{0}, \xi\right)$ or $A_{n}(\xi)$ then the solution is completely independent of time and is equivalent to a steady-state solution of (6.1). For the examples considered in $\S 5$, this implies that either

$$
\phi_{m}=\text { const, } \quad \text { or } \quad z_{r}=\text { const }, \quad \forall \xi .
$$

The steady-state solutions for a homogeneous, reverse graded and normally graded inflow into a semi-infinite chute are effectively illustrated in figures (6)-(8), where the $t$-axis must now be replaced by $x / u_{0}$. Another way to see this is that when $\partial / \partial t=0$, equation (6.1) reduces to

$$
u_{0} \frac{\partial \phi}{\partial x}-S_{r} \frac{\partial}{\partial z}(\phi(1-\phi))=D_{r} \frac{\partial^{2} \phi}{\partial z^{2}},
$$

which can be transformed to (5.3) with the mapping $x=u_{0} t$. The general solution given by (5.35)-(5.40) therefore holds with $t$ replaced by $x / u_{0}$.

\subsection{Time-dependent evolution towards a steady state}

Solutions that show the time-dependent evolution of the homogeneous, reverse graded or normally graded solutions towards the steady state in a semi-infinite chute can be constructed by setting

$$
\begin{aligned}
& \left.t_{s}=0, \quad \phi_{m}=0.55 \text { or } z_{r}=0.5 \text { for } \xi \geqslant 0,\right\} \\
& \left.t_{s}=-\xi / u_{0}, \quad \phi_{m}=0.55 \text { or } z_{r}=0.5 \text { for } \xi<0,\right\}
\end{aligned}
$$

where the parameters $\phi_{m}$ or $z_{r}$ are equal to the same constant for all columns. For columns $\xi \geqslant 0$, the time-shift parameter $t_{s}=0$, which implies that there is no lateral 
variation and the solution is purely time-dependent. For columns $\xi<0$, the time-shift parameter $t_{s}=-\xi / u_{0}$, which implies that the solution is at steady state, as shown in $\S 6.1$. The solution therefore consists of two zones: a region that was initially in the chute that is purely time-dependent; and a steady-state region created by the columns that enter into the chute. This is illustrated for the initially homogeneous solution in figure 9 for $D_{r}=0.1$ and $S_{r}=1$. Initially, all the material in the chute is at the homogeneous concentration $\phi_{m}=0.55$ and once the particles are released the small particles percolate downwards and lever the large particles upwards. The no normal flux condition (5.5) together with the incompatibility with the initial conditions (5.41) creates a diffuse, but rapidly thickening, layer of large particles at the free surface, and a layer of fines at the base, as shown for $t=0.1,0.2,0.3$. This drives the time-dependent solution rapidly towards steady state, which is attained locally at $t=2$. The interface between the laterally uniform time-dependent solution and spatial steady state lies at the kink in the contours. Since $u_{0}=1$, its position is the same as the time $t$ in each of the panels. Note, that Gray \& Thornton (2005) have constructed the corresponding solution for no-diffusive remixing in their figure 6.

Figure 10 shows the corresponding solutions for a reverse graded initial state with $D_{r}=0.1$ and $S_{r}=1$. Initially, the particles are sharply normally graded with all the fines on top of the coarse grains and separated by a concentration shock at $z_{r}=0.5$. The discontinuity is rapidly diffused away, so that significant concentrations of large particles reach the free surface by $t=0.2$. This is much quicker than in the no-diffusive remixing solution constructed by Thornton et al. (2006), where the large particles reach the free-surface only at $t=0.5$. This shows that the remixing process can help the particles to approach a segregated state more quickly than the non-diffusive case. By $t=0.5$, the large particles arriving at the free surface produce a diffuse coarse-grain-rich layer that grows in size with increasing time. By $t=1.5$, the solution is at steady state in half of the domain and it reaches a local steady state in $x \in(0,3)$ at $t=3$. Again the position of transition between the steady state and laterally uniform region is given by the time $t$, since $u_{0}=1$.

\subsection{Solutions for a time-varying inflow in a semi-infinite chute}

The final example shows some solutions in which there are periodic sinusoidal pulses, which may give some insight into what to expect when this occurs experimentally. This time

$$
\left.\begin{array}{lll}
t_{s}=0, & \phi_{m}=0.55 & \text { for } \quad \xi \geqslant 0 \\
t_{s}=-\xi / u_{0}, & \phi_{m}=0.55+0.1 \sin \left(10 t_{s}\right) & \text { for } \quad \xi<0 .
\end{array}\right\}
$$

The solution is illustrated in figure 11 for $D_{r}=0.1$ and $S_{r}=1$. For the grains that were initially in the chute, the time-shift is zero and the columns all have the same value of $\phi_{m}=0.55$, so the solution is identical to the laterally uniform time-dependent part of the solution illustrated in figure 9. However, the region behind the transition is not at steady state, because each of the columns has different values of the mean concentration $\phi_{m}$ dependent on when they entered the chute. The mean concentration is determined by the sinusoidal variation in (6.11), which in turn determines the final steady-state solution (3.4) that a fixed column will tend towards. Since the columns are moving downstream, the concentration in a fixed frame of reference varies in both space and time. Sufficiently far downstream, the solutions in each column are close to their steady state and in a fixed frame of reference this appears as propagating sinusoidal oscillations in the concentration contours. Note, that Gray \& Thornton (2005) have constructed the corresponding case with no diffusion (see their figure 7). 


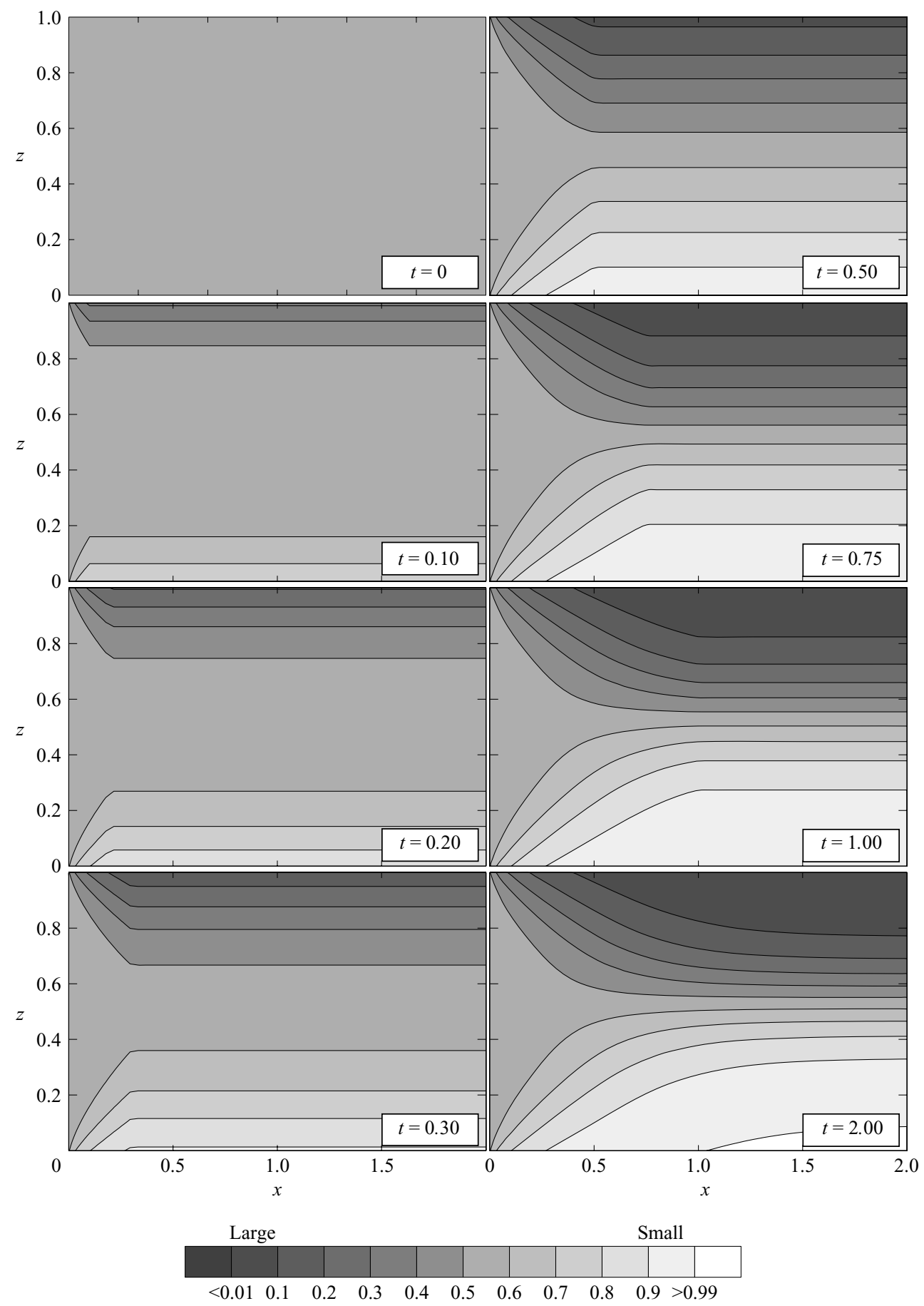

FIGURE 9. A sequence of contour plots showing the temporal evolution of the small-particle concentration $\phi$ as a function of $(x, z)$ for segregation and diffusive remixing in plug-flow. The bulk flow is from left to right with velocity $u_{0}=1$ and initially the avalanche is homogeneously mixed with mean concentration $\phi_{m}=0.55$. At $x=0$, the avalanche is fed with homogeneously mixed grains of the same concentration $\phi_{m}$. The segregation number $S_{r}=1$ and the diffusive remixing number $D_{r}=0.1$. A local steady state is attained at $t=2$. Note that the $x$-axis may be considerably elongated in the physical domain because of the differential vertical and horizontal scalings in (2.19). 

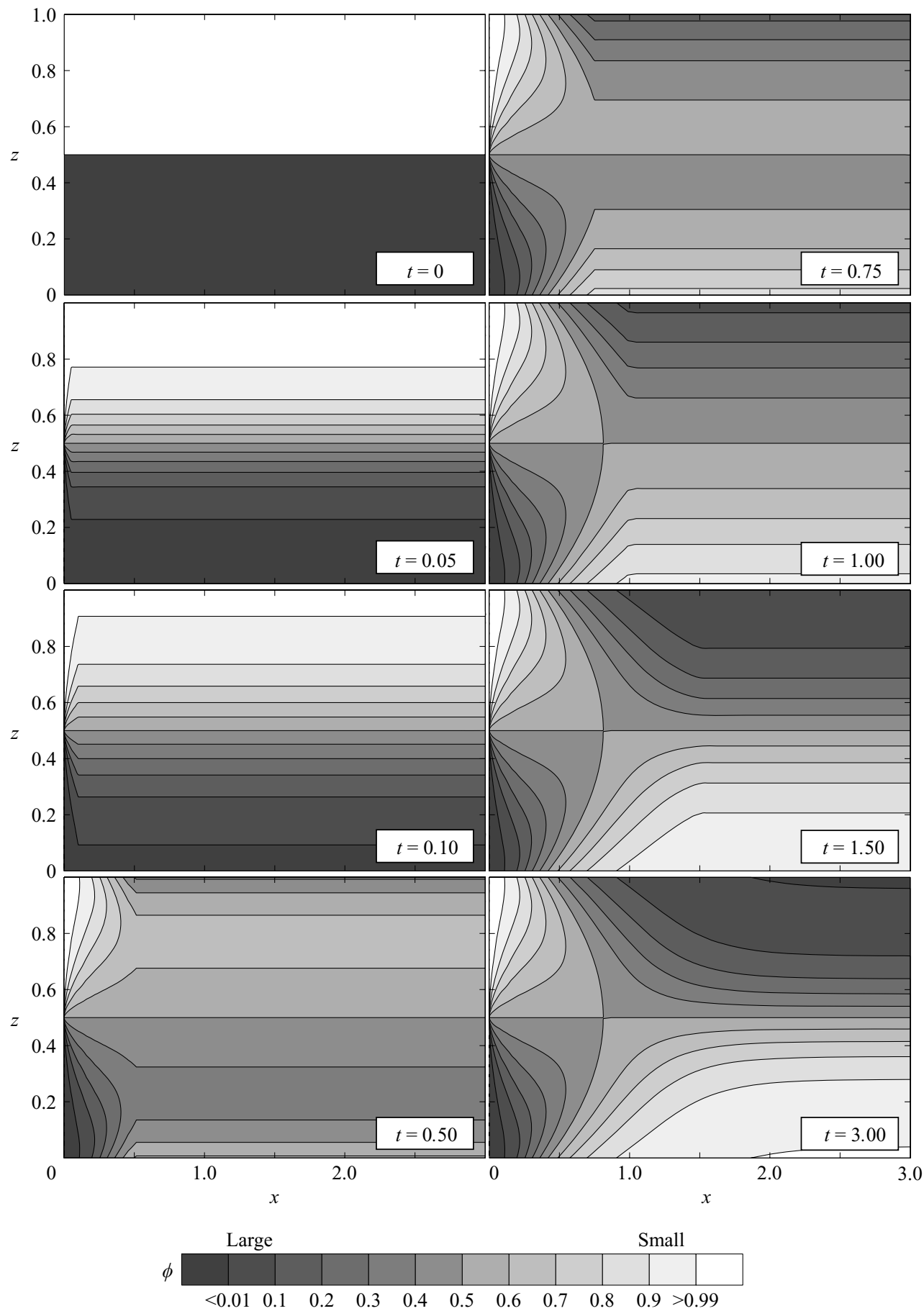

FIGURE 10. A sequence of contour plots showing the temporal evolution of the small-particle concentration $\phi$ as a function of $(x, z)$ for segregation and diffusive remixing in plug-flow. The bulk flow is from left to right with velocity $u_{0}=1$ and initially the avalanche is sharply normally graded at a height $z_{r}=0.5$, with all the small particles on top of the coarse grains. At $x=0$, the avalanche is fed with normally graded material with a jump in concentration at $z=z_{r}$. The segregation number $S_{r}=1$ and the diffusive remixing number $D_{r}=0.1$. A local steady state is attained at $t=3$. 


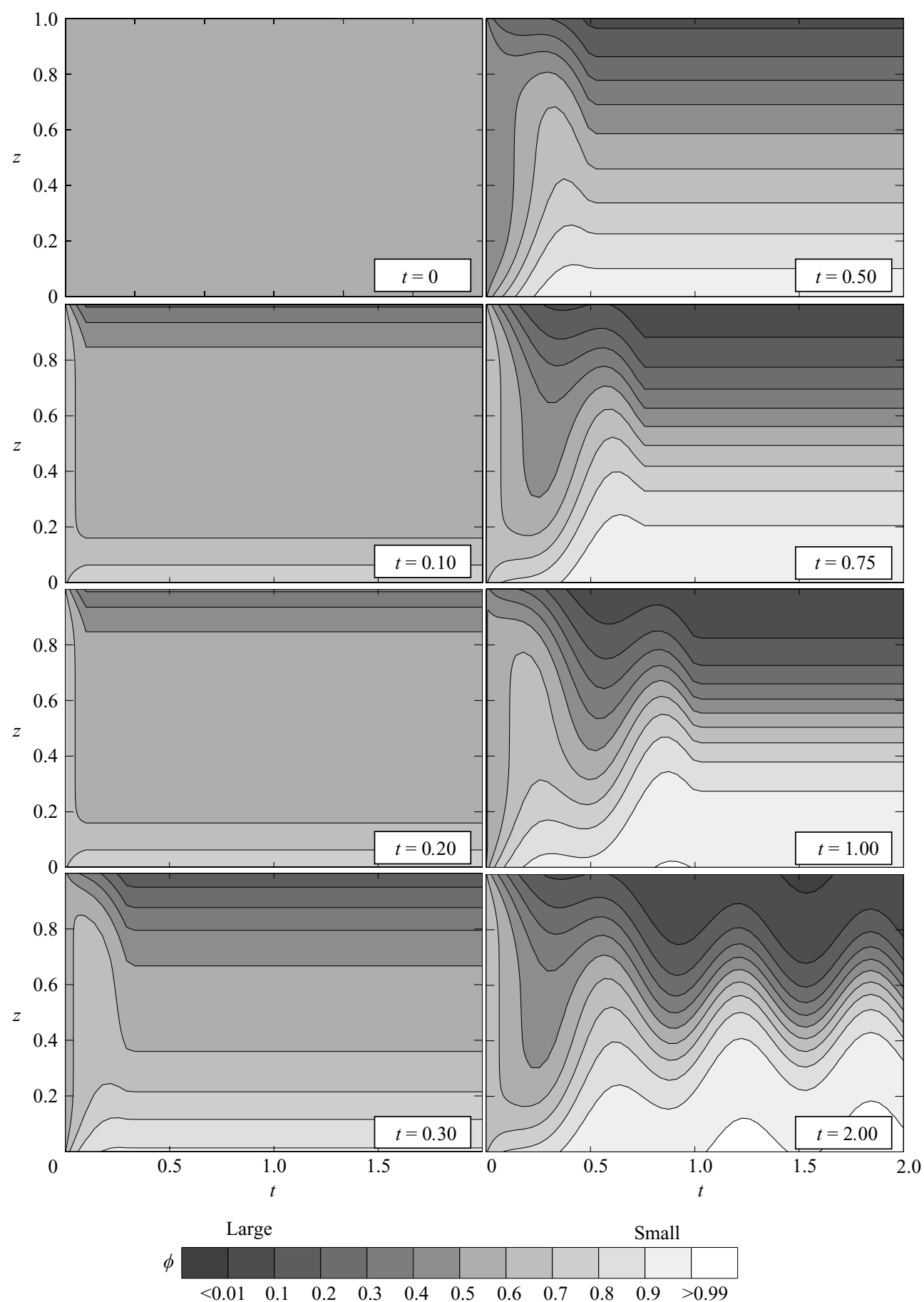

FIGURE 11. A sequence of contour plots showing the temporal evolution of the small-particle concentration $\phi$ as a function of $(x, z)$ for segregation and diffusive remixing in plug-flow. The bulk flow is from left to right with velocity $u_{0}=1$ and initially the avalanche is homogeneously mixed with mean concentration $\phi_{m}=0.55$. At $x=0$, the avalanche is fed with homogeneously mixed grains whose mean concentration varies sinusoidally in time with an amplitude of 0.1 around the background concentration of 0.55 . The segregation number $S_{r}=1$ and the diffusive remixing number $D_{r}=0.1$. 
Both solutions rely that the columns uncoupling in plug-flow. When there is shear with depth, the sinusoidal oscillations will be sheared over one another, causing further segregation and remixing.

\section{Discussion and conclusions}

This paper uses binary mixture theory to derive a model for particle-size segregation and diffusive remixing in an avalanche composed of large and small particles. The theory is a generalization of Gray \& Thornton's (2005) model for segregation by kinetic sieving and uses constituent momentum balances (2.3) to formulate equations for the normal percolation velocities (2.15) of the large and small grains. This provides a natural way of introducing gravity and diffusive remixing into the theory and ensures that the model is consistent with the gravity-driven nature of the kinetic sieving mechanism. This is a significant advantage over other segregation theories (e.g. Savage \& Lun 1988; Dolgunin \& Ukolov 1995) which are independent of gravity. The theory is designed to be compatible with the assumptions of shallowness, incompressibility and bulk lithostatic pressure, that are made by almost all current avalanche models (e.g. Grigorian et al. 1967; Kulikovskii \& Eglit 1973; Eglit 1983; Savage \& Hutter 1989; Iverson 1997; Gray et al. 1999, 2003; Iverson \& Denlinger 2001; Gray 2001). The segregation-remixing theory can therefore be coupled to these models to compute segregation and remixing in dynamically evolving avalanches. This opens up the possibility of coupling the bulk motion to the local size distribution through the modification of the pore-pressure or the basal friction to investigate the instabilities that these can generate (e.g. Pouliquen, Delour \& Savage 1997; Pouliquen \& Vallance 1999; Iverson \& Vallance 2001; Iverson 2003).

Since the boundary conditions (2.24) imply no flux of particles across the free surface, or the base, the steady-state solution to the segregation-remixing equation (2.20) is dependent on the initial mean concentration of small particles, as well as the segregation-remixing length scale $z_{0}$. This is defined in (3.5) as $z_{0}=D_{r} / S_{r}$ and shows that there is a competition between the effects of remixing and segregation. For low values of $z_{0}$, there is rapid smooth transition from high concentrations of fines to high concentrations of coarse particles with increasing height. For large $z_{0}$ the distribution is almost linear with a weak grading of the particles. Elementary solutions for laterally uniform time-dependent flows have also been constructed by using the Cole-Hopf transformation (5.10) to obtain a linear PDE that could be solved by standard methods. This allows us to write a general-solution (5.35)-(5.40) for arbitrary initial conditions $\phi_{0}(z)$. Three specific solutions are investigated when the initial concentration is (i) homogeneous, (ii) reverse graded, and, (iii) normally graded. These show that, for low $z_{0}$, the shocks, in the pure segregation solutions obtained by Gray \& Thornton (2005) and Thornton et al. (2006), are replaced by rapid smooth transitions. In the homogeneous problem, the boundary conditions are not compatible with the initial conditions, this drives a rapid readjustment to the solution that becomes increasingly strong with larger $z_{0}$. In plug-flow, the segregation and remixing in each material column is independent of that taking place in adjacent columns. As a result, it is possible to construct time-dependent two-dimensional solutions for plug-flow in a semi-infinite chute. These show that if the boundary conditions at the inflow are held constant, a spatial steady state develops. If they are varied, oscillatory solutions can be generated. The time-dependent and plugflow segregation-remixing solutions constructed in this paper are considerably more general and complex than the corresponding pure segregation solutions investigated 
by Gray \& Thornton (2005) and Thornton et al. (2006). However, the pure segregation limit is a very useful approximation when the diffusive remixing is small, as it is possible to construct steady-state and fully dynamic solutions (Gray et al. 2006) that take account of shear through the avalanche depth. There are also situations, such as in modelling the formation of patterns, when the simplicity afforded by the pure segregation limit gives us a two-tone picture that is easier to understand than the full solution.

This research was generously supported by a NATO Collaborative Linkage Grant PST.CLG.979600. J.M.N. T.G. also gratefully acknowledges the support of an EPSRC Advanced Research Fellowship (GR/S50052/01 and GR/S50069/01) as well as MIMS support from the School of Mathematics at the University of Manchester.

\section{REFERENCES}

BAGNOLD, R. A. 1954 Experiments on a gravity-free dispersion of large spheres in a Newtonian fluid under shear. Proc. R. Soc. Lond. A 255, 49-63.

Bear, J. 1972. Dynamics of Fluids in Porous Media. Elsevier.

Billingham, J. \& King, A. C. 2000 Wave Motion. Cambridge University Press.

Branney, M. J. \& KokelaAR, P. 1992 A reappraisal of ignimbrite emplacement: progressive aggradation and changes from particulate to non-particulate flow during emplacement of high-grade ignimbrite. Bull. Volcanol. 54, 504-520.

Bridgwater, J. 1976 Fundamental powder mixing mechanisms. Powder Technol. 15, 215-236.

Campbell, C. S. 2006 Granular material flows. An overview. Powder Technol. 162, 208-229.

Cantwell, B. J. 2002 Introduction to Symmetry Analysis. Cambridge University Press.

Chadwick, P. 1976 Continuum Mechanics. Concise Theory and Problems. Allen \& Unwin (republished Dover 1999).

Cole, J. D. 1951 On a quasilinear parabolic equation occurring in aerodynamics. Q. Appl. Maths $\mathbf{9}$, 225-236.

Dolgunin, V. N. \& Ukolov, A. A. 1995 Segregation modelling of particle rapid gravity flow. Powder Technol. 83, 95-103.

Dolgunin, V. N., Kudy, A. N. \& Ukolov, A. A. 1998 Development of the model of segregation of particles undergoing granular flow down an inclined chute. Powder Technol. 96, 211-218.

Drahun, J. A. \& Bridgwater, J. 1983 The mechanisms of free surface segregation. Powder Technol. 36, 39-53.

EGLit, M. E. 1983 Some mathematical models of snow avalanches. In Advances in Mechanics and the Flow of Granular Materials (ed. M. Shahinpoor), vol. 2, pp. 577-588. Clausthal-Zellerfeld and Gulf.

Ehrichs, E. E., Jaeger, H. M., Karczmar, G. S., Knight, J. B., Kuperman, V. Y. \& Nagel, S. R. 1995 Granular convection observed by magnetic-resonance-imaging. Science 267, 1632-1634.

GraY, J. M. N. T. 2001 Granular flow in partially filled slowly rotating drums. J. Fluid. Mech. 441, $1-29$.

Gray, J. M. N. T. \& Hutter, K. 1997 Pattern formation in granular avalanches. Contin. Mech. Thermodyn. 9, 341-345.

Gray, J. M. N. T. \& Hutter, K. 1998 Physik granularer Lawinen. Phys. Blätter 54, 37-43.

Gray, J. M. N. T. \& SvendSEN, B. 1997 Interaction models for mixtures with application to phase transitions. Intl J. Engng Sci. 35, 55-74.

Gray, J. M. N. T. \& TAI, Y. C. 1998 Particle size segregation, granular shocks and stratification patterns. NATO ASI series, Physics of Dry Granular Media (ed. H. J. Herrmann, J.-P. Hovi \& S. Luding), 697-702. Kluwer.

Gray, J. M. N. T. \& Thornton, A. R. 2005. A theory for particle size segregation in shallow granular free-surface flows. Proc. R. Soc. Lond. A 461, 1447-1473.

Gray, J. M. N. T., Wieland, M. \& Hutter K. 1999 Free surface flow of cohesionless granular avalanches over complex basal topography. Proc. R. Soc. A 455, 1841-1874. 
Gray, J. M. N. T., TaI, Y.-C. \& Noelle, S. 2003. Shock waves, dead-zones and particle-free regions in rapid granular free surface flows. J. Fluid. Mech. 491, 161-181.

Gray, J. M. N. T., Shearer, M. \& Thornton, A. R. 2006 Time-dependent solutions for particle-size segregation in shallow granular avalanches. Proc. R. Soc. Lond. A 462, 947-972.

Grigorian, S. S., EgLit, M. E. \& IAKImov, IU. L. 1967 New statement and solution of the problem of the motion of snow avalanche. In Snow, Avalanches and Glaciers. Tr. Vysokogornogo Geofizich Inst. Vol. 12.

HajRa, S. K \& Khakhar, D. V. 2004 Sensitivity of segregation of mixtures in quasi-two-dimensional fluidized layers. Phys. Rev. E 69, 031304.

Hopf, E. 1950 The partial differential equation $u_{t}+u u_{x}=\mu u_{x x}$. Commun. Pure Appl. Maths 3, 201-230.

Hsiau, S. S. \& Hunt, M. L. 1993 Shear-induced particle diffusion and longitudinal velocity fluctuations in a granular-flow mixing layer. J. Fluid Mech. 251, 299-313.

IVERSON, R. M. 1997 The physics of debris flows. Rev. Geophys. 35, 245-296.

Iverson, R. M. 2003 The debris-flow rheology myth. In Debris-Flow Hazards Mitigation: Mechanics, Prediction and Assessment (ed. Rickenmann \& R. C. Chen), pp. 303-314. Millpress, Rotterdam.

Iverson, R. M. \& Denlinger, R. P. 2001 Flow of variably fluidized granular masses across three-dimensional terrain 1. Coulomb mixture theory. J. Geophys. Res. 106, B1, 553-566.

Iverson, R. M. \& Vallance, J. W. 2001 New views of granular mass flows. Geology 29, 115-118.

JENKInS, J. T. 1998 Particle segregation in collisional flows of inelastic spheres. In Physics of Dry Granular Media (ed. H. J. Herrmann, J.-P. Hovi \& S. Luding), pp. 645-658. NATO ASI series, Kluwer.

JEnkins, J. T. \& MAncini, F. 1987 Balance laws and constitutive relations for plane flows of a dense, binary mixture of smooth nearly elastic, circular disks. J. Appl. Mech. 54, 27-34.

Jenkins, J. T. \& YoOn, D. 2001 Segregation in binary mixtures under gravity. $P R L$ 88(19), 194301-4.

Johanson, J. R. 1978 Particle segregation ... and what to do about it. Chem. Engng 85, 183-188.

Khakhar, D. V., McCarthy, J. J. \& Ottino, J. M. 1997 Radial segregation of granular mixtures in rotating cylinders. Phys. Fluids 9, 3600-3614.

Khakhar, D. V., McCarthy, J. J. \& Ottino, J. M. 1999 Mixing and segregation of granular materials in chute flows. Chaos 9, 594-610.

KulikovskiI, A. G. \& Eglit, M. E. 1973 Two-dimensional problem of the motion of a snow avalanche along a slope with smoothly changing properties. Prik. Mat. Mekh. 37, 837-848. (Transl. J. Appl. Maths Mech. 37, 792-803.)

Louge, M. Y. 2003 Model for dense granular flows down bumpy inclines. Phys. Rev. E 67, 061303$1-11$.

Middleton, G. V. \& Hampton, M. A. 1976 Subaqueous sediment transport and deposition by sediment gravity flows. In Marine Sediment Transport and Environmental Management (ed. D. J. Stanley \& D. J. P. Swift), pp. 197-218, Wiley.

Möbius, M. E., Lauderdale, B. E., Nagel, S. R. \& JAEGer, H. M. 2001 Size segregation of granular particles. Nature 414, 270.

Morland, L. W. 1992 Flow of viscous fluids through a porous deformable matrix. Surveys Geophys. 13, 209-268.

Pouliquen, O. \& Vallance, J. W. 1999 Segregation induced instabilities of granular fronts. Chaos 9, 621-630.

Pouliquen, O., Delour, J. \& Savage, S. B. 1997 Fingering in granular flows. Nature, 386, 816-817.

Savage, S. B. \& DAI, R. 1993 Studies of granular shear flows. Wall slip velocities, 'layering' and self-diffusion. Mech. Mat. 16, 225-238.

Savage, S. B. \& Hutter, K. 1989 The motion of a finite mass of granular material down a rough incline. J. Fluid Mech. 199, 177-215.

Savage, S. B \& Lun, C. K. K. 1988 Particle size segregation in inclined chute flow of dry cohesionless granular solids. J. Fluid Mech. 189, 311-335.

Sethian, J. A. 1999 Level set methods. and fast marching methods. Cambridge University Press.

Silbert, L. E., Ertas, D., Grest, G. S., Haley, T. C. Levine, D. \& Plimpton, S. J. 2001 Granular flow down an inclined plane: Bagnold scaling and rheology. Phys. Rev. E. 64, 051302.

Thomas, N. 2000 Reverse and intermediate segregation of large beads in dry granular media Phys. Rev. E 62, 961-974. 
Thornton, A. R., Gray, J. M. N. T. \& HogG, A. J. 2006 A three-phase mixture theory for particle size segregation in shallow granular free-surface flows. J. Fluid Mech. 550, 1-25.

Truesdell, C. 1984 Rational Thermodynamics. Springer.

Vallance, J. W. \& Savage, S. B. 2000 Particle segregation in granular flows down chutes. In IUTAM Symposium on segregation in granular materials (ed. A. D. Rosato \& D. L. Blackmore), pp. 31-51, Kluwer.

Whitham G. B. 1974 Linear and Nonlinear Waves. Wiley-Interscience.

Wills B. A. 1979 Mineral Processing Technology. Pergamon.

Xu, H., Louge, M. \& Reeves, A. 2003 Solutions of the kinetic theory for bounded collisional granular flows. Continuum Mech. Thermodyn. 15, 321-349.

Zhang, Y. \& ReESE, J. M. 2000 The influence of the drag force due to the interstitial gas on granular flows down a chute. Intl J. Multiphase Flow 26, 2049-2072. 